\title{
Long-term culture of fetal monocyte precursors in vitro allowing the generation of bona fide alveolar macrophages in vivo
}

\author{
Fengqi Li ${ }^{1, \#}$, Katarzyna Maria Okreglicka ${ }^{1, \#}$, Lea Maria Pohlmeier ${ }^{1}$, Christoph Schneider ${ }^{1,2}$, \\ Manfred Kopf ${ }^{1, *}$ \\ ${ }^{1}$ Institute of Molecular Health Sciences, Department of Biology, ETH Zürich, Switzerland. \\ ${ }^{2}$ Institute of Physiology, University of Zürich, Switzerland. \\ \#These authors contributed equally to this work. \\ *Correspondence author: Tel: +41 44633 64 70; E-mail: manfred.kopf@ethz.ch
}

Running title: A novel model for pulmonary macrophage transplantation therapy 


\begin{abstract}
Tissue-resident macrophage-based immune therapies have been proposed for various diseases. However, generation of sufficient numbers that possess tissue-specific functions remains a major handicap. Here, we show that fetal liver monocytes (FLiMo) cultured with GM-CSF (also known as CSF2) rapidly differentiate into a long-lived, homogeneous alveolar macrophage (AM)-like population in vitro. CSF2-cultured FLiMo remain the capacity to develop into bona fide AM upon transfer into Csf $2 \mathrm{ra}^{-/-}$neonates and prevent development of alveolar proteinosis and efferocytosis of apoptotic cells for at least 1 year in vivo. Compared to transplantation of AM-like cells derived from bone marrow macrophages (BMM), CSF2cFliMo more efficiently engraft empty AM niches in the lung and protect mice from respiratory viral infection. Harnessing the potential of this approach for gene therapy, we restored a disrupted Csf2ra gene in FLiMo and their capacity to develop into AM in vivo. Together, we provide a novel platform for generation of immature AM-like precursors amenable for genetic manipulation, which will be useful to study to dissect AM development and function and pulmonary transplantation therapy.
\end{abstract}

Key words: Alveolar macrophages, fetal liver monocytes, influenza virus, macrophage transplantation therapy 


\section{Introduction}

Tissue-resident macrophages (MФтR) are heterogeneous cell populations, present in almost all tissues and play multiple tissue-specific functions in homeostasis and diseases (Davies et al, 2013; Hoeffel \& Ginhoux, 2015). MФ-based therapies have been proposed as potential strategies in various diseases (Duan \& Luo, 2021; Mass \& Lachmann, 2021; Moroni et al, 2019; Peng et al, 2020).

Lung resident AM play important roles in host defense to pulmonary infections and noninflammatory clearance of inhaled particles (Hussell \& Bell, 2014). Moreover, AM are required for catabolism of surfactant and removal of apoptotic cells and cellular debris that otherwise accumulate in the alveoli resulting in impaired air exchange (Kopf et al, 2015). AM are derived from late EMP and fetal liver monocytes, which colonize the lung during late embryogenesis, and are maintained life-long by self-renewal after birth (Hashimoto et al, 2013; Yona et al, 2013). Development and expansion are driven perinatally by GM-CSFinduced PPAR $\gamma$ (Guilliams et al, 2013; Schneider et al, 2014). Accordingly, absence of PPAR $\gamma$, GM-CSF or one of the GM-CSFR subunits, Csf2ra and Csf2rb, in mice results in abrogated AM development and pulmonary alveolar proteinosis (PAP) (Guilliams et al, 2013; Nishinakamura et al, 1995; Robb et al, 1995; Schneider et al, 2017; Schneider et al, 2014). Moreover, human hereditary pulmonary alveolar proteinosis (herPAP) mutations, which is associated with long-term respiratory insufficiency and high susceptibility to microbial infection, is caused by mutations in CSF2RA and CSF2RB genes (Suzuki et al, 2011; Suzuki et al, 2008; Trapnell et al, 2003).

It has been shown that pulmonary transplantation of AM-like cells from BMM or induced pluripotent stem cells (iPSC) from yolk sac primitive macrophages or human blood $\mathrm{CD}^{+} 4^{+}$cells to $\operatorname{Cs} f 2 \mathrm{rb}^{-/-}$mice can prevent development of PAP (Happle et al, 2018; Happle et al, 2014; Lachmann et al, 2015; Mucci et al, 2018; Suzuki et al, 2014; Takata et al, 2017). 
However, whether these transplanted MФ resemble bona fide self-renewing AM with functional capacities beyond surfactant clearance remains unclear. Here, we have established a biologically relevant model to study AM development and function by gene editing in vitro and assessment of functional consequences in vivo. By culturing purified fetal liver monocytes with GM-CSF, we were able to generate high numbers of AM-like cells that can be kept proliferating like stem cells for several months in vitro and possess the ability to terminally differentiate and restore AM development and function upon pulmonary transplantation to $C s f 2 \mathrm{ra}^{--}$neonates. CSF2-cFLiMo-derived AM resemble bona fide AM in gene expression profile, surface markers, and functional capacities including potent selfrenewal capacity, clearance of surfactant, and efferocytosis of apoptotic cells during influenza virus infection. In contrast to BMM, CSF2-cFliMo more efficiently engraft empty AM niches and protect mice from influenza infection. Finally, CSF2RA re-expression in Csf $2 \mathrm{ra}^{-/}$FLiMo by retroviral gene transfer restored their potential to develop into bona fide AM. Altogether, this platform provides a potent platform to study genes involved in AM development and function, as well as for therapeutic approaches.

\section{Results}

In vitro GM-CSF-differentiated fetal liver monocytes give rise to self-renewing cells with AM-like phenotype

The fetal liver contains several myeloid precursors including primitive macrophages (pMФ) and EMP-derived monocytes (Gomez Perdiguero et al, 2015; Hoeffel et al, 2015). Recently, we and others have shown that the latter are the most potent precursors of AM (Li et al, 2020; van de Laar et al, 2016). To identify and better characterize the AM precursor, we sorted populations of viable $\mathrm{F} 4 / 80^{\text {lo }} \mathrm{CD} 11 \mathrm{~b}^{\text {int }} \mathrm{Ly} 6 \mathrm{C}^{+}$monocytes and viable $\mathrm{F} 4 / 80^{\text {hi }} \mathrm{CD} 11 \mathrm{~b}^{\text {lo }}$ pMФ from the fetal liver of E14.5 C57BL/6 embryos (Fig. E1A) and cultured them with GM-CSF 
in vitro (Fig. 1A). Monocytes grew exponentially, whereas $\mathrm{pM} \Phi$ disappeared within 2 weeks (Fig. E1B). At day 3 of the monocyte culture, a new population with macrophage characteristics $\left(\mathrm{F} 4 / 80^{+} \mathrm{Ly}^{-} \mathrm{C}^{-}\right.$cells $)$emerged, which quickly predominated and appeared to be homogenous after 9 days in the culture (Fig. 1B). The monocyte-to-macrophage differentiation was paralleled by an increase in cell granularity (SSC) and expression of the surface markers CD11c and Siglec-F (Fig. E1C). Similar phenotypic changes also occur during fetal monocyte to AM differentiation in the lung, as described previously (Guilliams et al, 2013; Schneider et al, 2014), although high expression of CD11b indicated that cultured cells did not fully complete AM differentiation (Fig. E1D) (Schneider et al, 2014). Notably, the AM-like phenotype was maintained for at least 4 months of continuous culture (Fig. E1D). From here on, we refer to $\mathrm{F} 4 / 80^{+} \mathrm{CD} 11 \mathrm{c}^{+} \mathrm{CD} 11 \mathrm{~b}^{+}$Siglec-F $\mathrm{F}^{+}$cells as $\mathrm{CSF} 2$-cultured fetal liver monocytes (CSF2-cFliMo). Similar results were obtained when CSF2-cFLiMo were generated from monocytes isolated from E16.5, E18.5, and E20.5 fetal livers (Fig. E1E). When differentiated CSF2-cFliMo cells were deprived of GM-CSF, their numbers rapidly declined (Fig. E1F, G), although the remaining cells maintained the expression of AM surface markers including CD11c and Siglec-F (Fig. E1H).

$\mathrm{TGF} \mathrm{R}^{-/-}$mice are defective in development of AM (Yu et al, 2017). Indeed, we found that addition of TGF $\beta$ increased the proliferative capacity and the yield of CSF2-cFLiMo, but did not influence the differentiation to AM-like cells (Fig. E2A, B). Notably, culture with TGF $\beta$ in the absence of GM-CSF failed to induce proliferation and resulted in death of FLiMo (Fig. E2B). Moreover, TGF $\beta$ did not change the phenotype of CSF2-cFliMo after 2week culture (Fig. E2C).

These results demonstrate that fetal liver monocytes cultured with GM-CSF in vitro give rise to a stable population of cells with AM-like phenotype and GM-CSF-dependent selfrenewing capacity. 


\section{CSF2-cFLiMo develop into mature and functional AM in vivo}

To assess whether CSF2-cFLiMo can develop to bona fide AM and perform AM function in vivo, we transferred congenically marked CSF2-cFLiMo intranasally (i.n.) to newborn Csf2 $r a^{-/}$mice (Fig. 1A), which lack AM (Li et al, 2020; Schneider et al, 2017). Analysis of the BAL and lung of Csf $2 \mathrm{ra}^{-/-}$recipients showed efficient engraftment of donor-derived cells that resemble mature CD11c ${ }^{\text {hi Siglec-F }}{ }^{\text {hi }}$ AM (Fig. 1C and Fig. E3A). The numbers of CSF2cFLiMo-derived AM rapidly increased within the first 6 weeks after transfer, before reaching a relatively stable population size (Fig. 1D), similar to the kinetics during normal postnatal AM differentiation (Guilliams et al, 2013; Schneider et al, 2014). While CSF2-cFLiMo were CD11b ${ }^{\text {hi }}$ Siglec-F $F^{\text {lo }}$ before transfer, they down-regulated CD11b and up-regulated CD11c and Siglec-F surface expression upon transfer and expansion in vivo, indicating that they completed their differentiation to become cells with a phenotype that is indistinguishable from AM of age-matched WT mice (Fig. 1E). Notably, CSF2-cFLiMo-derived AM were maintained in the lung for at least 1 year after transfer (Fig. 1C, D). Moreover, measurement of protein concentration in the BAL at different time points after transfer showed that CSF2cFLiMo-AM reconstituted $C s f 2 \mathrm{ra}^{-/}$mice were completely protected from PAP up to one year (Fig. 1F and Fig. E3B). These results demonstrate that CSF2-cFLiMo develop into mature AM, which appear functionally equivalent to in situ differentiated AM.

\section{CSF2-cFLiMo-derived AM can self-renew in vivo}

AM are maintained locally through self-renewal, and they are largely independent of adult hematopoiesis at steady state (Guilliams et al, 2013; Schneider et al, 2014). Serial transplantation remains the gold standard for experimental assessment of long-term repopulating and self-renewal capacity of hematopoietic stem cells (Iscove \& Nawa, 1997), 
but similar experiments have not been done for tissue macrophages. To determine the longterm self-renewing capacity of CSF2-cFLiMo-AM, we serially transferred in vitro differentiated CSF2-cFLiMo or ex vivo isolated AM from adult WT mice into neonatal Csf $2 \mathrm{ra}^{-/}$mice. After 6 weeks, donor-derived AM were isolated and transferred into a second group of neonatal Csf2 $\mathrm{ra}^{-/-}$recipients (Fig. 2A). Following secondary transfer, CSF2cFLiMo-AM and ex vivo AM-derived AM (AM-AM) again fully restored the AM compartment of $C s f 2 \mathrm{ra}^{-/}$recipients and their number and phenotype was comparable to that of $\mathrm{AM}$ in the BAL and lung of unmanipulated WT mice (Fig. 2B-D). These results demonstrate that CSF2-cFLiMo-AM have a self-renewal capacity in vivo, which is comparable to bona fide AM.

\section{CSF2-cFLiMo acquire AM-specific transcriptional signature in vivo}

To reveal the gene expression programs that are associated with particular AM differentiation stages, we compared the transcriptomes of CSF2-cFLiMo, CSF2-cFLiMo-derived AM to ex vivo AM and E14.5 fetal liver monocytes (Fig. 3A). Principal-component analysis (PCA) and matrix clustering, based on all detected genes, revealed that CSF2-cFLiMo-derived AM displayed substantial different gene expression profiles compared to fetal liver monocytes, and CSF2-cFLiMo, while they were closely related and clustered together with ex vivo AM (Fig. 3B-C). CSF2-cFLiMo-AM and ex vivo AM expressed low levels of several well-known monocyte markers, including Ly6c1 (Ly-6C), Fcgr1 (CD64), Itgam (CD11b) (Fig. 3D). Moreover, the relative mRNA expression of several established AM markers, including Marco, Pparg, Itgax (CD11c), MerTk, Cd14, Fcgr2b (CD32), Siglec5 (Siglec-F) and Chil3 (Ym1) was similar between CSF2-cFLiMo-AM and ex vivo AM (Fig. 3D).

Next, we compared differentially expressed genes (DEG) (fold change > 2) to signature genes defined for monocytes and lung macrophages (Lavin et al, 2014). More than $60 \%$ of 
monocyte signature genes were downregulated, while $60 \%$ of AM signature genes were upregulated in in fetal liver monocytes cultured with GM-CSF in vitro (Fig. E4A), suggesting that these AM signature genes are directly or indirectly regulated by GM-CSF. Interestingly, the remaining 40\% AM signature genes were up-regulated in CSF2-cFLiMo upon transfer and maturation in vivo (Fig. E4B).

The in vitro culture of precursors followed by in vivo transfer described here creates a model to study GM-CSF and other niche factors during AM development. Comparison of the CSF2-cFLiMo and fetal liver monocyte transcriptomes revealed 3301 upregulated and 2657 downregulated genes, which were dependent on GM-CSF (Fig. 3E). Similarly, comparing the transcriptomes of CSF2-cFLiMo-AM to CSF2-cFLiMo, we found 2032 genes that were upregulated and 1813 genes that were down-regulated by niche factors (Fig. 3E). The representative genes of the top 100 DEG regulated by CSF2 or niche factors are listed in Fig. 3F. Only a minor fraction of CSF2-upregulated genes was further upregulated (11.9\%) or downregulated $(20.3 \%$ ) by niche factors (Fig. 3G). Similarly, $7.8 \%$ and $8.8 \%$ of CSF2downregulated genes were further downregulated or upregulated by niche factors, respectively (Fig. 3G). These results showed that the majority of genes were separately regulated by $\mathrm{CSF} 2$ and additional niche factors. More than $60 \%$ of the gene expression changes were driven by CSF2 (Fig. 3G), indicating its major contribution to AM development.

Taken together, our results show that culture of fetal liver monocytes with GM-CSF in vitro results in AM-like precursors, which can accomplish full AM differentiation driven by alveolar niche factors upon intranasal transfer to AM-deficient neonates.

\section{CSF2-cFLiMo-derived AM are functional in homeostasis and during infection}


Overall, our studies demonstrate that CSF2-cFLiMo-AM were functionally equivalent to naturally differentiated AM. To determine the number of donor cells required to fully reconstitute the AM compartment of $C s f 2 \mathrm{ra}^{-/}$mice, we titrated the number of transferred CSF2-cFLiMo (Fig. 4A). Transfer of a minimum of $5 \times 10^{4}$ CSF2-cFLiMo to neonatal Csf $2 \mathrm{ra}^{-}$ ${ }^{1}$ mice resulted in AM numbers in adult recipients that were comparable to unmanipulated WT mice (around $5 \times 10^{5}$ ) (Fig. 4B) and protected mice from PAP (Fig. 4C). We have previously established that around $10 \%$ of primary fetal liver monocytes supplied intranasally reach the lung (Li et al, 2020). Thus, CSF2-cFLiMo have expanded around 100-fold 6 weeks after transfer to Csf $2 \mathrm{ra}^{-/-}$neonates. Notably, extended time of CSF2-cFLiMo in vitro culture (i.e. 4 months) prior transfer into recipient mice did not negatively affect their differentiation and functional capacity (Fig. 4B, C). Another critical function of tissue-resident macrophages including AM is the removal of apoptotic cells (efferocytosis) (Morioka et al, 2019). We compared efferocytosis between CSF2-cFLiMo-AM in Csf $2 \mathrm{ra}^{-/-}$mice and AM in WT mice by intratracheal (i.t.) installation of labelled apoptotic thymocytes. CSF2-cFLiMo-AM and AM were equally potent at phagocytosing apoptotic cells from the bronchoalveolar space (Fig. 4D).

Next, we assessed whether CSF2-cFLiMo show therapeutic activity upon transfer into adult $C s f 2 \mathrm{ra}^{-/-}$mice, which had already developed PAP. Adult $C s f 2 \mathrm{ra}^{-/-}$mice were transferred i.t. with $0.5,1$ or 2 million CSF2-cFLiMo (Fig. 4E-G). Ten weeks after transfer, donorderived AM were detectable in the BAL and lung of $C s f 2 \mathrm{ra}^{-/}$only in recipients transferred with 2 million cells (Fig. 4F). The protein levels in the BAL from mice transferred with $2 \times 10^{6}$ cells were significantly lower when compared to naïve $C s f 2 \mathrm{ra}^{-/-}$mice, suggesting that transferred cells were able to reduce proteinosis, although not to the level of WT mice (Fig. 4G). However, CSF2-cFLiMo-derived AM exhibited higher expression of F4/80 and CD11b, and lower expression of Siglec-F and CD64 when compared to WT AM (Fig. E5A, B), 
indicating that the AM phenotype was not fully recapitulated but intermediate between AMderived from CSF2-cFLiMo transferred to neonates and AM-derived from CSF2-cFLiMo transplanted to adult mice. These results show that CSF2-cFLiMo can reproduce AM phenotype and function most adequately only when transferred to neonatal $C s f 2 \mathrm{ra}^{-/}$mice.

In addition to the homeostatic function, AM play an essential role in protecting influenza virus-infected mice from morbidity by maintaining lung integrity through the removal of dead cells and excess surfactant (Schneider et al, 2014). To assess the functional capacity of CSF2-cFLiMo-derived AM during pulmonary virus infection, we reconstituted Csf2 $\mathrm{ra}^{-/-}$neonates with CSF2-cFLiMo and infected adults 10 weeks later with influenza virus PR8 (Fig. 5A). Without transfer, Csf $2 \mathrm{ra}^{-/-}$mice succumbed to infection due to lung failure (Fig. 5B-E), as reported previously (Schneider et al, 2017). Notably, the presence of CSF2cFLiMo-derived-AM protected $C s f 2 \mathrm{ra}^{-/}$mice from severe morbidity (Fig. 5B, C) and completely restored viability (Fig. 5D) and $\mathrm{O}_{2}$ saturation (Fig. 5E) compared to infected WT mice.

Recent studies have shown that transplantation of BMM could decrease proteinosis in adult $C s f 2 r b^{-/}$mice in homeostasis (Happle et al, 2014; Lachmann et al, 2014; Suzuki et al, 2014). To directly compare the capacity of BMM and CSF2-cFLiMo in AM development, we transferred M-CSF-derived BMM and CSF2-cFLiMo at a 1:1 ratio into neonatal Csf $2 \mathrm{ra}^{-/}$ recipient mice (Fig. 5G). Analysis AM compartment 10 weeks after transfer showed that more than $70 \%$ were derived from CSF2-cFLiMo (Fig. 4H, I) and that their phenotype $\left(\mathrm{CD} 11 \mathrm{~b}^{\text {lo }}\right.$ Siglec- $\left.\mathrm{F}^{\mathrm{hi}}\right)$ closely resembled that from genuine AM, while the remaining $30 \%$ BMM-derived AM showed differences in CD11b, Siglec-F, and CD64 surface levels (Fig. 4J, K). Similar results were shown when transferring GM-CSF-derived BMM and CSF2-cFLiMo at a 1:1 ratio into neonatal $C s f 2 \mathrm{ra}^{-/-}$recipient mice (Fig. 5G). However, transfer of BMM to 
Csf $2 \mathrm{ra}^{-/}$neonates in the absence of competing CSF2-cFLiMo resulted in AM numbers comparable to resident $\mathrm{AM}$ in untreated mice (Fig. 5F). To study the function of BMMderived AM during pulmonary virus infection, we infected $C s f 2 \mathrm{ra}^{-/-}$recipients containing BMM-AM with influenza virus PR8 (Fig. 5A). In contrast to CSF2-cFLiMo-transferred Csf $2 \mathrm{ra}^{--}$mice, the presence of BMM-derived-AM was of no advantage and mice succumbed to infection comparable to $C s f 2 r a^{\lrcorner}$mice devoid of AM (Fig. 5B-E). These results indicate that CSF2-cFLiMo-derived AM but not BMM-derived AM protect from influenza-induced morbidity and mortality.

\section{Major and minor histocompatibility differences of CSF2-cFLiMo result in transplant rejection}

Our results suggest that CSF2-cFLiMo could be used as an elegant in vitro and in vivo system to study the development and function of AM. To gain more information about the potential of this system, we next evaluated the MHC compatibility in an allogeneic CSF2-cFLiMo transfer. We generated CSF2-cFLiMo from BALB/c E14.5 embryos and transferred them alone, or together with C57BL/6 (B6) CSF2-cFLiMo at a 1:1 ratio into neonatal Csf2ra mice that were on B6 background (Fig. 6A). Ten weeks later, we were unable to detect any BALB/c CSF2-cFLiMo-AM, regardless if they had been transferred alone or together with B6 CSF2-cFLiMo, indicating that complete rejection of MHC-mismatched cells (Fig. 6B, C and Fig. E6A, B). We then also compared Y chromosome compatibility by transferring GMCSF-differentiated monocytes isolated from male neonatal (day 0-3 after birth) liver (CSF2cNLiMo) to newborn $C s f 2 \mathrm{ra}^{-/}$male or female recipients (Fig. 6D). Donor-derived AM could be detected in all transferred animals regardless of recipient's sex (Fig. 6E, F). However, female recipients displayed significantly lower AM numbers compared to male recipients, which indicates that there was only a minor Y chromosome-dependent selection. Together, 
our data indicate that both major histocompatibility complex and minor histocompatibility antigens have to be considered to avoid rejection of AM grafts.

\section{Gene therapy of Csf2ra mutant CSF2-cFLiMo}

The reconstitution of AM by transferring cultivatable precursors provides unique opportunities for genetic manipulation. To provide proof-of-concept, fetal liver monocytes were purified from E14.5 Csf2 $\mathrm{ra}^{-/-}$or WT embryos and transduced with a retrovirus encoding Csf2ra-gfp (RV $\left.\mathrm{RV}^{C s f 2 r a-g f p}\right)$ or control $g f p$ only $\left(\mathrm{RV}^{g f p}\right)\left(\right.$ Fig. 7A). $\mathrm{RV}^{C s f 2 r a-g f p}$ transduced Csf2radeficient FLiMo expanded in the presence of GM-CSF in vitro (Fig. 7B) and outgrew all non-transduced cells, as indicated by the presence of almost $100 \% \mathrm{GFP}^{+}$cells by day 7 of culture (Fig. 7B, C). As expected, Csf2ra-deficient FLiMo transduced with control RV ${ }^{g f p}$ could not expand in culture (Fig. 7B, C). Notably, overexpression of Csf2ra in WT FLiMo provided also a slight growth advantage over non-transduced WT FLiMo in vitro (Fig. 7B, C). Next, we transferred $\mathrm{RV}^{C s f 2 r a-g f p}$ transduced $C s f 2 r a$-deficient cFLiMo into neonatal $\operatorname{Cs} f 2 \mathrm{ra}^{-/-}$mice and analyzed BAL and lung 8 weeks later. Indeed, CSF2RA gene therapy of Csf2ra-deficient cFLiMo enabled full reconstitution of a functional AM compartment that prevented development of PAP in $C s f 2 \mathrm{ra}^{-/}$mice (Fig. 7D-G). Transfer of Csf2raoverexpressing WT cFLiMo did not result in higher AM numbers indicating that GM-CSF bioavailability rather than receptor expression is the limiting factor. $\mathrm{RV}^{C s f 2 r a-g f p}$ transduced cFLiMo-derived AM were phenotypically indistinguishable from unmanipulated WT AM for multiple surface markers, including F4/80, CD11b, CD11c, Siglec-F, and MHCII (Fig 7H). These results provide a proof-of-concept that gene-modified CSF2-cFLiMo differentiate into functional bona fide AM, therefore allowing genetic manipulation of this important tissue macrophage compartment. 


\section{Discussion}

We and others have previously shown that transfer of wild-type fetal liver or fetal lung monocytes can restore defective AM development in Csf2ra-deficient or Csf2rb-deficient mice (Guilliams et al, 2013; Li et al, 2020; Schneider et al, 2017; Schneider et al, 2014; van de Laar et al, 2016). In the present study, we describe a two-step AM differentiation model, which in a first step allows the generation of a homogenous and stable immature AM-like population (i.e. CSF2-cFLiMo) from murine fetal liver monocytes that can be maintained long-term in culture in the presence of GM-SF. In a second step, upon pulmonary transplantation, CSF2-cFLiMo

expand and complete differentiation to bona fide AM. Indeed, low numbers of CSF2-cFLiMo completely restored the AM pool of Csf2ra-deficient mice within 6 weeks and were stably maintained for at least 1 year, indicating that they efficiently occupied empty AM niches and possess a long-term self-renewing capacity, which we have proven by serial transplantation. While serial transplantation is the gold standard for proof of long-term repopulation and selfrenewal capacity of hematopoietic stem cells, according to our knowledge, to date it has not been applied to tissue macrophages.

Studies in knockout mice have shown that GM-CSF and TGF- $\beta$ are essential for AM development mainly by induction of the transcription factor PPAR $\gamma$ and its target genes (Guilliams et al, 2013; Schneider et al, 2014; Yu et al, 2017). Our FLiMo in vitro cultures suggest that GM-CSF is sufficient for AM growth and differentiation of AM precursors, while TGF- $\beta$ further promotes their proliferative capacity. TGF- $\beta$ alone was insufficient to grow FLiMo in vitro. Notably, it has been suggested that fetal liver cells can be grown longterm in the presence of GM-CSF in vitro (Fejer et al, 2013). However, in contrast to fetal liver monocytes, fetal liver macrophages, which are derived from primitive yolk sac 
macrophages, could not be expanded in vitro, despite the presence of Csf2ra and Csf2rb chains, consistent with their poor capacity to restore AM compartment upon neonatal transfer to Csf2ra-deficient mice (Li et al, 2020).

Even though FLiMo upregulated a large panel of AM signature genes in the presence of GM-CSF in vitro, PCA analysis of the transcriptome showed a difference to that of mature AM. However, upon pulmonary transfer, CSF2-cFLiMo completed differentiation into bona fide AM indicating that lung tissue instructs terminal differentiation of AM. Thus, the twostep model allows to separate the transcriptional regulation induced by GM-CSF from other niche factors provided by the lung microenvironment during AM development. Further studies using this model will help to understand transcriptional regulation during AM development.

Notably, when CSF2-cFLiMo were transplanted to the lung of adult Csf2ra-deficient recipients, Siglec-F upregulation and $\mathrm{CD} 11 \mathrm{~b}$ downregulation occurred less efficiently as compared to pulmonary transplantation to neonates, indicating that CSF2-cFLiMo transplantation is more efficient in neonates than in adults. We can think of two possible reasons. Firstly, the occurrence of proteinosis in adult $C s f 2 r a^{-/-}$mice might obstruct the contact between transplanted cells and lung epithelial cells, which is important for the survival and engraftment of transplanted cells. Secondly, the neonatal lung niche could more efficiently promote AM maturation compared to the adult lung niche. Thus, the macrophage transplantation treatment for hereditary PAP patients would be more efficient during the neonatal or childhood stage before development of severe proteinosis. Importantly, we showed that the lung alveolus is not an immune privileged site implicating that transplantation AM-like precursors to MHC- and ever gender-mismatched recipients results in rejection. Thus, our data indicate that both MHC and Y chromosome compatibility require consideration when transferring CSF2-cFLiMo precursors. 
M-CSF-derived BMM have been widely used to study macrophage biology in vitro, although they poorly recapitulate the heterogenous phenotypic and functional features of genuine resident macrophage subsets that are present in every tissue. Despite, M-CSFderived BMM or GM-CSF-derived BMM can differentiate to AM upon pulmonary transfer to $C s 2 r b$-deficient mice (Suzuki et al, 2014). Furthermore, induced pluripotent stem cell (iPSC)-derived macrophages originating from primitive macrophages in the mouse yolk sac or from human blood $\mathrm{CD} 34^{+}$cells have been proposed as models for pulmonary macrophage transfer therapies (Buchrieser et al, 2017; Mucci et al, 2018; Takata et al, 2017). So far, the capacity to prevent alveolar proteinosis has served as the only functional parameter for quality control in all of these approaches, except in one study that included phagocytosis of bacteria (Neehus et al, 2018).

AM develop from fetal monocytes independent of yolk sac primitive macrophages and bone marrow precursors in steady state (Hoeffel et al, 2015; Yona et al, 2013). Several studies showed recently that AM derived from primitive yolk-sac derived macrophages or from bone marrow monocytes are different in metabolism and function (Aegerter et al, 2020; Gibbings et al, 2015; Machiels et al, 2017; Misharin et al, 2017). In fact, comparing AM derived from either CSF2-cFLiMo or BMM, we found that the latter possess impaired capacity to engraft, expand, and acquire a bona fide AM phenotype and function including protection of $C s f 2 \mathrm{ra}^{-/-}$mice from mortality and respiratory failure during influenza infection. These results demonstrate that catabolism of accumulated proteins in the BAL is insufficient to assess the efficacy of pulmonary macrophage transplantation therapies and that GM-CSF cultured FLiMo are superior to AM-like cells derived from bone marrow or blood cultures. CSF2-cFLiMo-derived AM prevented PAP, performed efferocytosis of apoptotic cells, and protected from fatal respiratory viral infection, indicating that they acquired the broad functional spectrum of bona fide AM. 
CSF2-cFLiMo generated from wild-type or gene-deficient mice could be used as a highthroughput screening system to study AM development in vitro and in vivo. Our model is suitable to study the relationship between AM and lung tissue, as well as the roles of specific genes or factors in AM development and function. Furthermore, CSF2-cFLiMo can overcome the limitation in macrophage precursor numbers and be used as a therapeutic approach for PAP disease or in other macrophage-based cell therapies including lung emphysema, lung fibrosis, lung infectious disease and lung cancer (Byrne et al, 2016; Lee et al, 2016; Wilson et al, 2010). Finally, genetically modified and transferred CSF2-cFLiMo might facilitate the controlled expression of specific therapeutic proteins in the lung for disease treatment, and therefore, could represent an attractive alternative to non-specific gene delivery by viral vectors.

\section{Materials and Methods}

\section{Mice}

C57BL/6 CD45.2, congenic CD45.1 and, BALB/c mice were originally from the Jackson Laboratory (Bar Harbor, Maine, USA). Csf2 $\mathrm{ra}^{-/-}$mice were established in our laboratory (Schneider et al, 2017). All mice were housed and bred under specific pathogen-free conditions in individually ventilated cages in a controlled day-night cycle at the ETH Phenomics Facility. Mice used for experiments were 6-10 weeks (adults) of age, unless otherwise stated. All animal experiments were performed according to the guidelines (Swiss Animal Protection Ordinance (TschV) Zurich) and Swiss animal protection law (TschG) and had been approved by the local animal ethics committee (Kantonales Veterinaersamt Zurich).

\section{Timed pregnancy}


Female C57BL/6 CD45.1, CD45.2, or BALB/c mice were housed together with matching male mice overnight. The vaginal plug was checked on the next day and was designated as embryonic day $0.5(\mathrm{E} 0.5)$.

\section{Pulmonary cell transplantation}

Neonatal (day 0-3 after birth) $C s f 2 r a^{-/-}$recipient mice were transferred intranasally (i.n.) with different numbers of cells in $10 \mu \mathrm{L}$ endotoxin-free PBS. For competitive transfer experiments, 25,000 cells from each origin were mixed and transferred. Adult Csf2ra ${ }^{-/}$ recipient mice were transferred intratracheally (i.t.) with different numbers of cells in $50 \mu \mathrm{L}$ endotoxin-free PBS.

\section{Cell suspension preparation}

Mice were euthanized by overdose (400 mg/kg body weight) of sodium pentobarbital by i.p. injection. The lungs were washed three times with $0.4 \mathrm{ml}$ of ice-cold PBS containing $2 \mathrm{mM}$ EDTA through an intratracheal cannula only when mice were older than 4 weeks. BAL fluid was collected and cells were harvested by centrifugation. Lungs were removed after perfusion with ice-cold PBS. Pregnant females were sacrificed by $\mathrm{CO}_{2}$ asphyxiation. Fetal livers were removed at the indicated time points. Organs were minced and then digested at 37 ${ }^{\circ} \mathrm{C}$ in IMDM medium containing $2.0 \mathrm{mg} / \mathrm{ml}$ of type IV collagenase (Worthington), 0.125 $\mathrm{mg} / \mathrm{ml}$ DNase I (Sigma) and 3\% FCS for 45 min (lungs) or 15 min (fetal and neonatal livers) respectively, and subsequently passed through a $70-\mu \mathrm{m}$-cell strainer (Becton Dickinson). Ammonium-chloride-potassium (ACK) lysing buffer was used for erythrocyte lysis for all samples.

\section{Flow cytometry and cell sorting}


Multiparameter assessment and cell sorting were performed using LSR Fortessa, BD FACS ARIA II and ARIA III (BD Biosciences) and data were analyzed with FlowJo software (TreeStar). After blocking the FcgIII/II receptors by incubation with homemade antiCD16/32 (2.4G2), single-cell suspensions were incubated with the indicated fluorochromeconjugated or biotinylated monoclonal antibodies in FACS buffer (PBS containing 2\% FCS and 2 mM EDTA) and then washed twice before detection. Monoclonal antibodies specific to mouse CD45 (30-F11), CD11c (N418), F4/80 (BM8), CD11b (M1/70), Siglec-F (E50-2440, BD Biosciences), CD45.1 (A20), CD45.2 (104), Ly6C (HK1.4), GM-CSFRa (698423, R\&D), CD64 (X54-5/7.1) and MHC class II (M5/114.15.2, eBioscience) were purchased from BioLegend unless otherwise stated. Dead cells were excluded using the live/dead marker eFluor780 (eBioscience).

\section{Generation of CSF2-cFLiMo}

Fetal livers were harvested from CD45.1 ${ }^{+}$C57BL/6 embryos. Liver single-cell suspensions were prepared. Monocytes were sorted using flow cytometry. Sorted monocytes were cultured in complete RPMI, supplemented with GM-CSF (PeproTech) (30 ng/mL) in vitro. Cells were plated with $1 \times 10^{5}$ cells $/ \mathrm{ml}$ and sub-cultured by splitting them 1:5 every 3 days. CSF2-cFLiMo used for transplantation were cultured for 2 weeks in culture media prior to transfers unless stated otherwise.

\section{Generation of BMM}

For the preparation of mouse BM-derived macrophages (BMM), tibias and femurs from the hind legs of adult donor mice were flushed with PBS. Bone marrow was rinsed through a 70um cell strainer (Becton Dickinson) followed by red blood cell depletion with ammoniumchloride-potassium (ACK) lysing buffer. BMM were differentiated in vitro in complete 
RPMI, supplemented with $10 \mathrm{ng} / \mathrm{ml}$ M-CSF (PeproTech) or 30ng/ml GM-CSF (PeproTech). Medium was replaced on day 3 and day 6. Adherent cells were harvested and used as mature BMM on day 7 or 8 .

\section{Assessment of total protein}

Total protein concentrations in BAL fluid were detected by Pierce BCA Protein Assay Kit according to the manufacturer's instructions (Thermo Scientific).

\section{RNA sequencing}

100,000 cells of indicated populations were collected into TRIzol (Life Technologies). Phase separation was achieved with the addition of chloroform (Sigma), and total RNA was precipitated from the aqueous layer with isopropanol (Sigma) using glycogen (Roche) as a carrier. RNA samples were sent to the Functional Genomics Center Zurich, where the RNA sequencing was performed. The TruSeq RNA Stranded sample kit (Illumina) was used to construct the sequencing libraries. In brief, total RNA samples (100 ng) were poly (A) enriched and reverse-transcribed into double-stranded cDNA, and TruSeq adapters were then ligated to double-stranded cDNA, then fragments containing TruSeq adapters on both ends were selectively enriched with PCR and subsequently sequenced on the Illumina Nova Seq. The fragments were mapped to the ensemble mouse reference genome GRCm38 (Version25.06.2015) using the STAR aligner (Dobin et al, 2013). For normalization, the read counts were scaled with the use of the trimmed mean of M-values (TMM) method proposed by Robinson and Oshlack (Robinson \& Oshlack, 2010). Principal-component analysis, matrix clustering and heatmap were generated using $\mathrm{R}$.

\section{Efferocytosis of apoptotic cells}


Thymocytes were isolated from mice and apoptosis was induced by exposure to $60 \mathrm{~mJ} / \mathrm{cm}^{2}$ UV radiation (Spectrolinker XL-1500; Spectronics Corporation). After $2 \mathrm{~h}$ incubation at 37 ${ }^{\circ} \mathrm{C}$ in IMDM $+10 \%$ FCS, cells were labeled with $5 \mathrm{mM}$ eFluor670 (eBioscience) according to the manufacturer's instructions, washed extensively with IMDM $+10 \%$ FCS and PBS. Then apoptotic cells ( 5 million cells in $50 \mu \mathrm{L}$ PBS) were delivered i.t. to recipient mice. 0.5, 2 and $22 \mathrm{~h}$ after administration, efferocytosis by AM in the BAL and lung was assessed by flow cytometry.

\section{Influenza viral infection}

Influenza virus strain PR8 (A/Puerto Rico/34, H1N1) was originally provided by J. Pavlovic, University Zurich. For infections, the mice were anesthetized and intratracheally (i.t.) inoculated with indicated doses of virus in 50 ul endotoxin-free PBS. Temperature and weight of mice were monitored daily and animals were euthanized if they fulfilled the severity criteria set out by institutional and cantonal guidelines.

\section{Measurement of oxygen saturation}

The MouseOx ${ }^{\mathrm{TM}}$ Puls-oximeter (Starr Life Sciences) was used to measure oxygen $\left(\mathrm{O}_{2}\right)$ saturation in influenza-infected mice on day 7 post infection. The depilatory agent was applied to the neck of mice 2-3 days prior to measurement to remove hair. Mice were sedated with $2.5 \mathrm{mg} / \mathrm{kg}$ intraperitoneal Midazolam (Roche) $0.5-1$ hour before measurement. The sensor clip was placed on the neck and $\mathrm{O}_{2}$ saturation was measured each second over 3-5 min per mouse. Data shown is the average value of each mouse.

\section{RV-reconstitution of CSF2RA gene}


Two retroviral constructs based on moloney murine leukemia virus, containing Csf2ra cDNA and GFP $\left(\mathrm{RV}^{C s f 2 r a-g f p}\right)$ or GFP only $\left(\mathrm{RV}^{g f p}\right)$, were used for transfection of retrovirus packaging cell line. Fresh viral supernatants containing non-replicating retroviruses were used for transduction of fetal liver monocytes isolated as described above from $C s f 2 \mathrm{ra}^{-/-}$or CD45.1 ${ }^{+}$ WT embryos. Cells were cultured in complete RPMI, supplemented with GM-CSF (30 $\mathrm{ng} / \mathrm{mL}$ ) for 7 days. All non-transduced fetal liver monocytes derived from $C s f 2 \mathrm{ra}^{-/-}$embryos could not proliferate. After a week, a homogenous population of receptor-expressing cells was detected and used for transfers. For WT cells, all live cells treated with the Csf2raoverexpressing virus were used (irrespectively of the transduction level).

\section{Statistical analysis}

Mean values and SEM were calculated with Prism (GraphPad Software, Inc). Student's t-test (unpaired) was used for comparing two groups, and ANOVA (one way) was used for comparing multiple groups: ns, not significant; $* \mathrm{p}<0.05$, **p $<0.01, * * * \mathrm{p}<0.001$, ****p $<$ 0.0001 .

\section{Acknowledgements}

We thank the teams of the ETH Flow Cytometry Core Facility for cell sorting and the EPIC mouse facility for animal husbandry, Peter Nielsen for discussion and editing of the manuscript. We are grateful for research grants from SNF (310030_163443 and 310030B_182829).

\section{Author contributions}


F.L., K.O., and M.K. designed the experiments; F.L. and K.O. performed and analyzed the experiments. L.P. analyzed RNA sequencing data. C.S. and M.K. discussed data and provided conceptualization. F.L., K.O., and M.K. wrote the manuscript. 


\section{References}

Aegerter H, Kulikauskaite J, Crotta S, Patel H, Kelly G, Hessel EM, Mack M, Beinke S, Wack A (2020) Influenza-induced monocyte-derived alveolar macrophages confer prolonged antibacterial protection. Nat Immunol 21: 145-157

Buchrieser J, James W, Moore MD (2017) Human Induced Pluripotent Stem Cell-Derived Macrophages Share Ontogeny with MYB-Independent Tissue-Resident Macrophages. Stem Cell Reports 8: 334-345

Byrne AJ, Maher TM, Lloyd CM (2016) Pulmonary Macrophages: A New Therapeutic Pathway in Fibrosing Lung Disease? Trends Mol Med 22: 303-316

Davies LC, Jenkins SJ, Allen JE, Taylor PR (2013) Tissue-resident macrophages. Nat Immunol 14: 986-995

Dobin A, Davis CA, Schlesinger F, Drenkow J, Zaleski C, Jha S, Batut P, Chaisson M, Gingeras TR (2013) STAR: ultrafast universal RNA-seq aligner. Bioinformatics 29: 15-21

Duan Z, Luo Y (2021) Targeting macrophages in cancer immunotherapy. Signal Transduct Target Ther 6: 127

Fejer G, Wegner MD, Gyory I, Cohen I, Engelhard P, Voronov E, Manke T, Ruzsics Z, Dolken L, Prazeres da Costa O et al (2013) Nontransformed, GM-CSF-dependent macrophage lines are a unique model to study tissue macrophage functions. Proc Natl Acad Sci U S A 110: E2191-2198

Gibbings SL, Goyal R, Desch AN, Leach SM, Prabagar M, Atif SM, Bratton DL, Janssen W, Jakubzick CV (2015) Transcriptome analysis highlights the conserved difference between embryonic and postnatal-derived alveolar macrophages. Blood 126: 1357-1366

Gomez Perdiguero E, Klapproth K, Schulz C, Busch K, Azzoni E, Crozet L, Garner H, Trouillet C, de Bruijn MF, Geissmann F et al (2015) Tissue-resident macrophages originate from yolk-sac-derived erythro-myeloid progenitors. Nature 518: 547-551

Guilliams M, De Kleer I, Henri S, Post S, Vanhoutte L, De Prijck S, Deswarte K, Malissen B, Hammad H, Lambrecht BN (2013) Alveolar macrophages develop from fetal monocytes that differentiate into long-lived cells in the first week of life via GM-CSF. J Exp Med 210: 19771992

Happle C, Lachmann N, Ackermann M, Mirenska A, Gohring G, Thomay K, Mucci A, Hetzel M, Glomb T, Suzuki T et al (2018) Pulmonary Transplantation of Human Induced Pluripotent Stem Cell-derived Macrophages Ameliorates Pulmonary Alveolar Proteinosis. Am J Respir Crit Care Med 198: 350-360

Happle C, Lachmann N, Skuljec J, Wetzke M, Ackermann M, Brennig S, Mucci A, Jirmo AC, Groos S, Mirenska A et al (2014) Pulmonary transplantation of macrophage progenitors as effective and long-lasting therapy for hereditary pulmonary alveolar proteinosis. Sci Transl Med 6: 250ra113 
Hashimoto D, Chow A, Noizat C, Teo P, Beasley MB, Leboeuf M, Becker CD, See P, Price J, Lucas D et al (2013) Tissue-resident macrophages self-maintain locally throughout adult life with minimal contribution from circulating monocytes. Immunity 38: 792-804

Hoeffel G, Chen J, Lavin Y, Low D, Almeida FF, See P, Beaudin AE, Lum J, Low I, Forsberg EC et al (2015) C-Myb(+) erythro-myeloid progenitor-derived fetal monocytes give rise to adult tissue-resident macrophages. Immunity 42: 665-678

Hoeffel G, Ginhoux F (2015) Ontogeny of Tissue-Resident Macrophages. Front Immunol 6: 486

Hussell T, Bell TJ (2014) Alveolar macrophages: plasticity in a tissue-specific context. Nat Rev Immunol 14: 81-93

Iscove NN, Nawa K (1997) Hematopoietic stem cells expand during serial transplantation in vivo without apparent exhaustion. Curr Biol 7: 805-808

Kopf M, Schneider C, Nobs SP (2015) The development and function of lung-resident macrophages and dendritic cells. Nat Immunol 16: 36-44

Lachmann N, Ackermann M, Frenzel E, Liebhaber S, Brennig S, Happle C, Hoffmann D, Klimenkova O, Luttge D, Buchegger T et al (2015) Large-scale hematopoietic differentiation of human induced pluripotent stem cells provides granulocytes or macrophages for cell replacement therapies. Stem Cell Reports 4: 282-296

Lachmann N, Happle C, Ackermann M, Luttge D, Wetzke M, Merkert S, Hetzel M, Kensah G, Jara-Avaca M, Mucci A et al (2014) Gene correction of human induced pluripotent stem cells repairs the cellular phenotype in pulmonary alveolar proteinosis. Am J Respir Crit Care Med 189: 167-182

Lavin Y, Winter D, Blecher-Gonen R, David E, Keren-Shaul H, Merad M, Jung S, Amit I (2014) Tissue-resident macrophage enhancer landscapes are shaped by the local microenvironment. Cell 159: 1312-1326

Lee S, Kivimae S, Dolor A, Szoka FC (2016) Macrophage-based cell therapies: The long and winding road. J Control Release 240: 527-540

Li F, Okreglicka KM, Pohlmeier LM, Schneider C, Kopf M (2020) Fetal monocytes possess increased metabolic capacity and replace primitive macrophages in tissue macrophage development. EMBO J 39: e103205

Machiels B, Dourcy M, Xiao X, Javaux J, Mesnil C, Sabatel C, Desmecht D, Lallemand F, Martinive P, Hammad $\mathrm{H}$ et al (2017) A gammaherpesvirus provides protection against allergic asthma by inducing the replacement of resident alveolar macrophages with regulatory monocytes. Nat Immunol 18: 1310-1320

Mass E, Lachmann N (2021) From macrophage biology to macrophage-based cellular immunotherapies. Gene Ther 
Misharin AV, Morales-Nebreda L, Reyfman PA, Cuda CM, Walter JM, McQuattie-Pimentel AC, Chen CI, Anekalla KR, Joshi N, Williams KJN et al (2017) Monocyte-derived alveolar macrophages drive lung fibrosis and persist in the lung over the life span. J Exp Med 214: 2387-2404

Morioka S, Maueroder C, Ravichandran KS (2019) Living on the Edge: Efferocytosis at the Interface of Homeostasis and Pathology. Immunity 50: 1149-1162

Moroni F, Dwyer BJ, Graham C, Pass C, Bailey L, Ritchie L, Mitchell D, Glover A, Laurie A, Doig S et al (2019) Safety profile of autologous macrophage therapy for liver cirrhosis. Nat Med 25: 1560-1565

Mucci A, Lopez-Rodriguez E, Hetzel M, Liu S, Suzuki T, Happle C, Ackermann M, Kempf H, Hillje R, Kunkiel J et al (2018) iPSC-Derived Macrophages Effectively Treat Pulmonary Alveolar Proteinosis in Csf2rb-Deficient Mice. Stem Cell Reports 11: 696-710

Neehus AL, Lam J, Haake K, Merkert S, Schmidt N, Mucci A, Ackermann M, Schubert M, Happle C, Kuhnel MP et al (2018) Impaired IFNgamma-Signaling and Mycobacterial Clearance in IFNgammaR1-Deficient Human iPSC-Derived Macrophages. Stem Cell Reports 10: 7-16

Nishinakamura R, Nakayama N, Hirabayashi Y, Inoue T, Aud D, McNeil T, Azuma S, Yoshida S, Toyoda Y, Arai K et al (1995) Mice deficient for the IL-3/GM-CSF/IL-5 beta c receptor exhibit lung pathology and impaired immune response, while beta IL3 receptordeficient mice are normal. Immunity 2: 211-222

Peng R, Ji H, Jin L, Lin S, Huang Y, Xu K, Yang Q, Sun D, Wu W (2020) MacrophageBased Therapies for Atherosclerosis Management. J Immunol Res 2020: 8131754

Robb L, Drinkwater CC, Metcalf D, Li R, Kontgen F, Nicola NA, Begley CG (1995) Hematopoietic and lung abnormalities in mice with a null mutation of the common beta subunit of the receptors for granulocyte-macrophage colony-stimulating factor and interleukins 3 and 5. Proc Natl Acad Sci U S A 92: 9565-9569

Robinson MD, Oshlack A (2010) A scaling normalization method for differential expression analysis of RNA-seq data. Genome Biol 11: R25

Schneider C, Nobs SP, Heer AK, Hirsch E, Penninger J, Siggs OM, Kopf M (2017) Frontline Science: Coincidental null mutation of Csf2ralpha in a colony of PI3Kgamma-/- mice causes alveolar macrophage deficiency and fatal respiratory viral infection. J Leukoc Biol 101: 367376

Schneider C, Nobs SP, Kurrer M, Rehrauer H, Thiele C, Kopf M (2014) Induction of the nuclear receptor PPAR-gamma by the cytokine GM-CSF is critical for the differentiation of fetal monocytes into alveolar macrophages. Nat Immunol 15: 1026-1037

Suzuki T, Arumugam P, Sakagami T, Lachmann N, Chalk C, Sallese A, Abe S, Trapnell C, Carey B, Moritz T et al (2014) Pulmonary macrophage transplantation therapy. Nature 514: 450-454 
Suzuki T, Maranda B, Sakagami T, Catellier P, Couture CY, Carey BC, Chalk C, Trapnell BC (2011) Hereditary pulmonary alveolar proteinosis caused by recessive CSF2RB mutations. Eur Respir J 37: 201-204

Suzuki T, Sakagami T, Rubin BK, Nogee LM, Wood RE, Zimmerman SL, Smolarek T, Dishop MK, Wert SE, Whitsett JA et al (2008) Familial pulmonary alveolar proteinosis caused by mutations in CSF2RA. J Exp Med 205: 2703-2710

Takata K, Kozaki T, Lee CZW, Thion MS, Otsuka M, Lim S, Utami KH, Fidan K, Park DS, Malleret B et al (2017) Induced-Pluripotent-Stem-Cell-Derived Primitive Macrophages Provide a Platform for Modeling Tissue-Resident Macrophage Differentiation and Function. Immunity 47: 183-198 e186

Trapnell BC, Whitsett JA, Nakata K (2003) Pulmonary alveolar proteinosis. N Engl J Med 349: 2527-2539

van de Laar L, Saelens W, De Prijck S, Martens L, Scott CL, Van Isterdael G, Hoffmann E, Beyaert R, Saeys Y, Lambrecht BN et al (2016) Yolk Sac Macrophages, Fetal Liver, and Adult Monocytes Can Colonize an Empty Niche and Develop into Functional TissueResident Macrophages. Immunity 44: 755-768

Wilson AA, Murphy GJ, Hamakawa H, Kwok LW, Srinivasan S, Hovav AH, Mulligan RC, Amar S, Suki B, Kotton DN (2010) Amelioration of emphysema in mice through lentiviral transduction of long-lived pulmonary alveolar macrophages. J Clin Invest 120: 379-389

Yona S, Kim KW, Wolf Y, Mildner A, Varol D, Breker M, Strauss-Ayali D, Viukov S, Guilliams M, Misharin A et al (2013) Fate mapping reveals origins and dynamics of monocytes and tissue macrophages under homeostasis. Immunity 38: 79-91

Yu X, Buttgereit A, Lelios I, Utz SG, Cansever D, Becher B, Greter M (2017) The Cytokine TGF-beta Promotes the Development and Homeostasis of Alveolar Macrophages. Immunity 47: 903-912 e904 


\section{Figure Legends}

Figure 1. Fetal liver monocytes can proliferate in vitro with GM-CSF and further develop into mature functional $\mathrm{AM}$ in vivo

(A) Illustration of experimental regime. Primary monocytes from fetal liver were cultured in vitro with GM-CSF. (B) Representative dot plots for F4/80 vs Ly6C are shown for GM-CSFcultured fetal liver monocytes (CSF2-cFLiMo) at the indicated time points, pre-gated on viable $\mathrm{CD}^{+} 5^{+}$single cells. (C-F) $5 \times 10^{4}$ CSF2-cFLiMo generated from CD45.1 E14.5 embryos, after 2 weeks of culturing, were transferred intranasally (i.n.) to neonatal (day 0-3 after birth) CD45.2 $2^{+} \mathrm{Cs} f 2 \mathrm{ra}^{-/-}$mice and analyzed at the indicated time points. (C) Representative dot plots of donor-derived cells in the bronchoalveolar lavage (BAL) of recipients 6 weeks, 6 months and 1 year after transfer, pre-gated as viable single cells. (D) Total numbers of donor-derived AM from lung (1 and 2 weeks) or from BAL and lung (after 6 weeks) detected in recipients at the indicated time-points. (E) Representative histograms of AM signature marker expression on CSF2-cFLiMo before transfer, CSF2-cFLiMo-derived $\mathrm{AM}$ detected in $\operatorname{Csf} 2 \mathrm{ra}^{-/}$recipient mice 6 weeks after transfer, and endogenous AM from age-matched control mice showing FMO control (grey) and specific antibodies against the indicated markers (black line). (F) Total protein in the BAL of CSF2-cFLiMo-transferred $C s f 2 \mathrm{ra}^{-/}$, unmanipulated $C s f 2 \mathrm{ra}^{-/-}$and WT mice. The data are representative of three independent experiments. Values show means \pm SEM of three to five mice per group in D and F. Student's t test (unpaired) was used in F: ns, not significant; *p $<0.05$, **p $<0.01$, $* * * \mathrm{p}<0.001, * * * * \mathrm{p}<0.0001$

\section{Figure 2. CSF2-cFLiMo-derived AM have ability to self-renew in vivo}

(A) Illustration of experimental regime. $5 \times 10^{4}$ of CD45.1 CSF2-cFLiMo generated from E14.5 embryos after 2-week culture or CD45.1 AM sorted from WT adult mice were 
transferred i.n. to neonatal CD45.2 $\operatorname{Cs} f 2 \mathrm{ra}^{-/-}$mice. After 6 weeks, donor-derived AM were sorted and $5 \times 10^{4}$ of cells were transferred i.n. to new neonatal $C s f 2 \mathrm{ra}^{-/-}$mice. BAL and lung were analysed 6 weeks after second-round transfer in B-D. (B) Representative dot plots showing the phenotype of CD45.1 donor-derived AM in the BAL and lung, pre-gated as viable $\mathrm{CD}^{4} 5^{+}$single cells. (C-D) Numbers of donor-derived AM and WT AM in the BAL (C) and lung (D). Age-matched $C s f 2 \mathrm{ra}^{-/-}$and CD45.2 WT mice were included as negative and positive controls, respectively. The data are representative of three experiments. Values show means \pm SEM of three to four mice per group. ANOVA (one way) was used in C-D: ns, not significant; $* \mathrm{p}<0.05, * * \mathrm{p}<0.01, * * * \mathrm{p}<0.001, * * * * \mathrm{p}<0.0001$

\section{Figure 3. Gene expression profiles of transferred CSF2-cFLiMo in $\mathrm{Cs}_{\mathrm{f}} \mathrm{ra}^{-/-}$mice}

(A) Illustration of experimental regime. Primary monocytes from E14.5 fetal liver were sorted using flow cytometry. CSF2-cFLiMo were collected after 2 weeks. CSF2-cFLiMo were transferred i.n. to neonatal $C s f 2 \mathrm{ra}^{-/-}$mice. Mice were analysed 6 weeks after transfer and CSF2-cFLiMo-derived mature AM (CSF2-cFLiMo-AM) were sorted from the BAL as viable $\mathrm{CD} 45^{+} \mathrm{CD} 11 \mathrm{c}^{\mathrm{hi}} \mathrm{F} 4 / 80^{+}$Siglec- $\mathrm{F}^{\mathrm{hi}} \mathrm{CD} 11 \mathrm{~b}^{\text {lo }}$ cells. AM from 6-week-old WT mice were sorted as control. RNA sequencing was performed (two biological replicates per group). (B) Principal component analysis (PCA) and (C) matrix clustering of the transcriptomes of all samples are shown. (D) Heat maps showing expression of monocyte and AM markers. (E) The numbers of upregulated and downregulated genes by CSF2 or niche. (F) Heat map showing the top 100 DEG and reprehensive genes of CSF2 and niche regulated. (G) Venn diagram of differentially expressed genes. Intersections of CSF2-upregulated or CSF2downregulated vs niche-upregulated or niche-downregulated genes. The absolute gene numbers and percentages in the intersections are shown. 


\section{Figure 4. CSF2-cFLiMo-derived AM are functional in phagocytosis and efferocytosis}

(A) Illustration of experimental regime. Different numbers of CD45.1 CSF2-cFLiMo after 2week $(2 \mathrm{w})$ or 4 -month $(4 \mathrm{~m})$ of culturing were transferred i.n to neonatal CD45.2 Csf2ra mice and analysed 6 weeks later in B-C. (B) Total numbers of donor-derived AM in the BAL and lung of recipient $C s f 2 \mathrm{ra}^{-/-}$mice or AM in the BAL and lung of WT mice. (C) Total protein levels in the BAL. (D) Efferocytosis of i.t. instilled apoptotic thymocytes by AM at the indicated time points. Values shown depict percentages of efferocytic AM. (E) Illustration of experimental regime. CD45.1 C57/BL6 CSF2-cFLiMo were generated from E14.5 embryos and cultured 2 weeks in vitro. Different numbers of CSF2-cFLiMo were transferred i.t. to 10 -week-old adult $C s f 2 \mathrm{ra}^{-/-}$mice and analysed 10 weeks later in F-G. (F) Total numbers of donor-derived AM in the BAL and lung of recipient $C s f 2 \mathrm{ra}^{-/-}$mice or AM in the BAL and lung of WT mice. $(\mathrm{G})$ Total protein levels in the BAL. Age-matched Csf2ra ${ }^{-/}$ and WT C57BL/6 mice were included as negative and positive controls, respectively. Values

show means \pm SEM and the results are representative of three experiments. Student's t test (unpaired) was used in D and ANOVA (one way) was used in B, C, F and G: ns, not significant; $* \mathrm{p}<0.05, * * \mathrm{p}<0.01, * * * \mathrm{p}<0.001, * * * * \mathrm{p}<0.0001$

Figure 5. CSF2-cFLiMo, but not BMM transplantation protects $\mathrm{Cs}_{2} 2 \mathrm{ra}^{-/-}$mice from respiratory failure following influenza infection.

(A) Illustration of experimental regime. CSF2-cFLiMo generated from E14.5 embryos after 2-week culture or BMM generated from adults after 7-day culture were i.n. transferred to neonatal CD45.2 $\mathrm{Cs} f 2 \mathrm{ra}^{-/}$mice. Adult recipient mice were infected i.t. with 10 pfu PR8 influenza virus and analysed in B-F. Shown are loss of body weight (B) and temperature (C), relative to the day of infection. (D) Survival curve showing the fraction of surviving animals for each day after infection. (E) Oxygen $\left(\mathrm{O}_{2}\right)$ saturation on 7 days p.i. is shown. $(\mathrm{F})$ Donor- 
derived AM numbers in the BAL and lung of $C s f 2 \mathrm{ra}^{-/-}$mice 8 weeks after AM reconstitution described in Fig. 5A and prior to infection. Age-matched $C s f 2 r a^{-/-}$and WT mice were included as negative and positive controls, respectively. (G) Illustration of experimental regime. CD45.1 CSF2-cFLiMo were generated from E14.5 embryos and cultured 2 weeks in vitro. CD45.1CD45.2 BMM were generated from adults and cultured 7 days with M-CSF or GM-CSF in vitro. CSF2-cFLiMo and BMM were pooled in 1:1 ratio and transferred i.n. to neonatal CD45.2 Csf2 $\mathrm{ra}^{-/-}$mice and analysed 10 weeks later in H-K. (H-I) Representative dot plots $(\mathrm{H})$ and percentage $(\mathrm{I})$ of donor-derived AM in BAL and lung of the recipients. $(\mathrm{J}-\mathrm{K})$ Representative histograms (J) and mean fluorescence intensity (MFI) (K) of AM signature markers on CSF2-cFLiMo- and BMM-derived AM and WT AM. Values show means \pm SEM and the results are representative of three experiments. Student's t test (unpaired) was used in I and ANOVA (one way) was used in E, F and K: ns, not significant; *p $<0.05,{ }^{*} \mathrm{p}<0.01$, $* * * \mathrm{p}<0.001, * * * * \mathrm{p}<0.0001$

\section{Figure 6. The MHC and gender compatibility in allogenic CSF2-cFLiMo transfers}

(A) Illustration of experimental regime. CD45.2 BALB/c and CD45.1 B6 CSF2-cFLiMo were generated from E14.5 embryos and cultured 2 weeks in vitro. BALB/c CSF2-cFLiMo were transferred to neonatal CD45.2 Csf $2 \mathrm{ra}^{-/-}$mice (B6 background) either separately or in a 1:1 ratio with B6 CSF2-cFLiMo and analysed 10 weeks later in B-C. (B) Representative dot plots showing the phenotype of donor-derived AM in the BAL, pre-gated on viable $\mathrm{CD} 45^{+}$ single cells. (C) Percentage of donor-derived AM in BAL of co-transferred recipients. (D) Illustration of experimental regime. CSF2-cNLiMo generated from CD45.1 WT male neonates after 2-week culture were intranasally transferred to neonatal CD45.2 Csf $2 \mathrm{ra}^{-/-}$mice respectively and analysed after 10 weeks in E-F. Mice were grouped according to gender. (EF) Numbers of donor-derived AM and WT AM in the BAL (E) and lung (F) are shown. Age- 
matched $C s f 2 \mathrm{ra}^{-/-}$and CD45.2 WT mice were included as negative and positive controls in B, $\mathrm{E}$ and $\mathrm{F}$. Values show means $\pm \mathrm{SEM}$ in $\mathrm{C}, \mathrm{E}$ and $\mathrm{F}$ and the results are representative of three experiments. Student's t test (unpaired) was used: ns, not significant; ${ }^{*} \mathrm{p}<0.05,{ }^{*} \mathrm{p}<0.01$, $* * * \mathrm{p}<0.001, * * * * \mathrm{p}<0.0001$.

Figure 7. Csf2ra-reconstituted $C_{s} f \mathrm{ra}^{-/-}$CSF2-cFLiMo can differentiate into functional AM in vivo

(A) Illustration of experimental regime. Fetal liver (FL) monocytes were purified from E14.5 CD45.2 Csf2 $\mathrm{ra}^{-/}$or CD45.1 WT embryos and spin infected with GM-CSFRa-GFP or GFP (control) retrovirus $\left(\mathrm{RV}^{C s f 2 r a-g f p}\right.$ or $\left.\mathrm{RV}^{g f p}\right)$. Cells were cultured with $\mathrm{CSF} 2$ for 7 days. $\mathrm{RV}^{C s f 2 r a-}$ $g f p$ transduced $C s f 2 \mathrm{ra}^{-/}$CSF2-cFLiMo $\left(\mathrm{RV}^{\text {Csf } 2 r a-g f p}\right.$-cFLiMo) or identically treated CD45.1 WT CSF2-cFLiMo were transferred i.n. to neonatal CD45.2 ${ }^{+} \mathrm{Csf} 2 \mathrm{ra}{ }^{-/-}$mice and evaluated after 8 weeks. (B) Efficiency of spin infection $\left(\mathrm{GFP}^{+}\right)$and survival of cultured cells at day 3 and 7 post-infection. (C) Expression levels of GM-CSFRa on $\mathrm{RV}^{C s f 2 r a-g f p}$ or RV ${ }^{g f p}$ transduced Csf2 $\mathrm{ra}^{-{ }^{-}}$or WT cFLiMo at day 3 and 7 post-infection. (D) Representative dot plots showing the phenotype of donor-derived cells in the BAL and lung, pre-gated on viable $\mathrm{CD}_{4} 5^{+}$single cells. (E-F) Numbers of donor-derived AM and WT AM in the BAL (E) and lung (F). (G) Total protein levels in the BAL are shown. Age-matched $C s f 2 \mathrm{ra}^{-/-}$and CD45.2 WT mice were included as negative and positive controls in D-G, respectively. (H) Representative histograms of AM signature marker expression, GM-CSFRa and GFP on RV $\mathrm{R}^{C s f r a-g f p_{-}}$ cFLiMo-derived AM and WT AM showing FMO control (grey) and specific antibodies against indicated markers (black line). The data are representative of three experiments. Values show means \pm SEM of three to four mice per group. Student's t test (unpaired) was used in E-G: ns, not significant; *p $<0.05, * * p<0.01, * * * p<0.001, * * * * p<0.0001$ 
bioRxiv preprint doi: https://doi.org/10.1101/2021.06.04.447115; this version posted June 8, 2021. The copyright holder for this preprint (which was not certified by peer review) is the author/funder. All rights reserved. No reuse allowed without permission. 


\section{Figure 1}

A

B In vitro

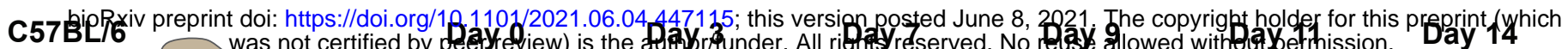
(CD45.1)

E14.5

Embryos
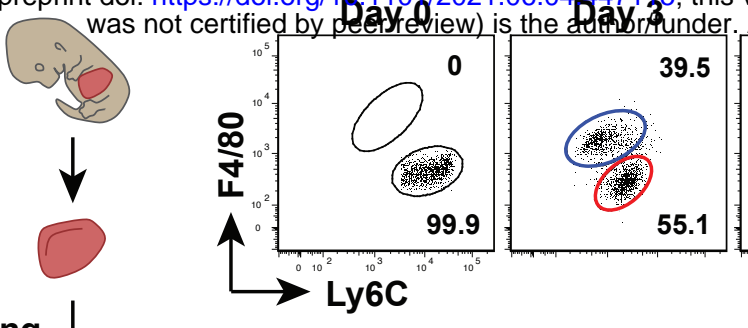

C

BAL

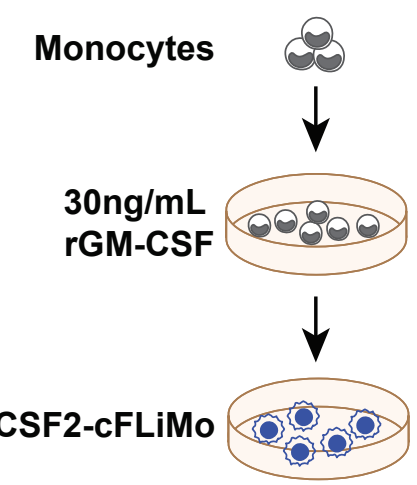

i.n. transfer $\downarrow$

Csf2ra-

Neonates

(CD45.2)
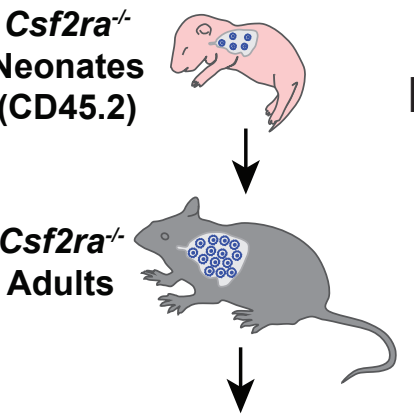

Analysis

(BAL and Lung)

D

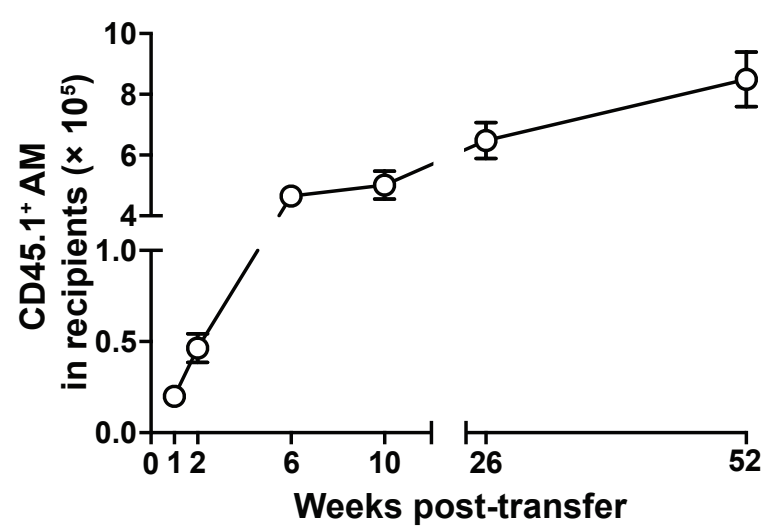

F

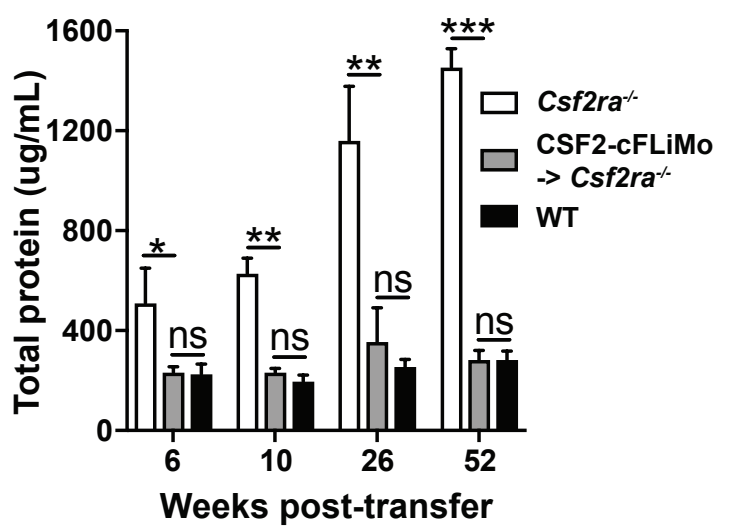

E
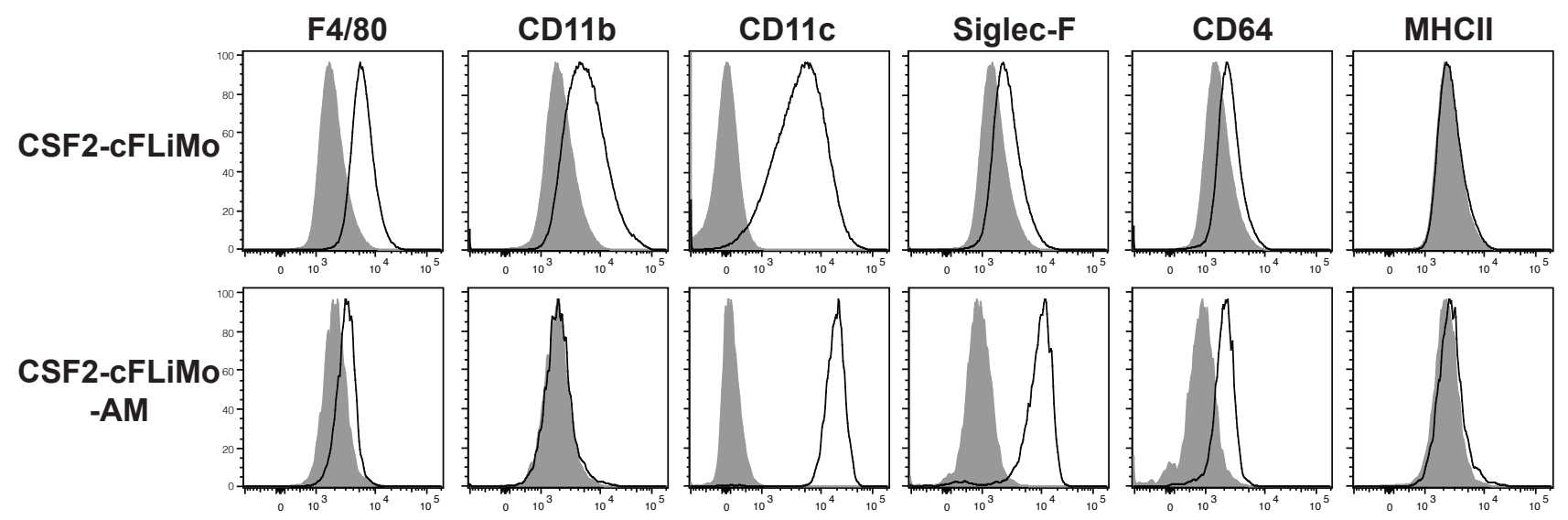

$\square$ Stained

FMO

WT AM
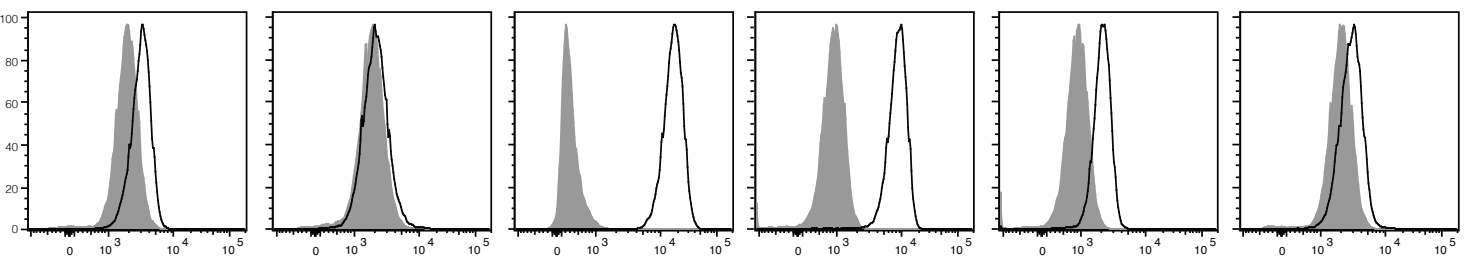


\section{Figure 2}

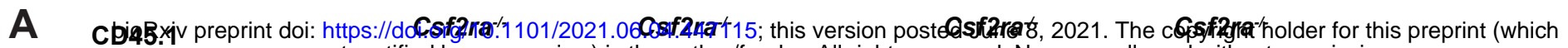
CSF2-cFLiMo

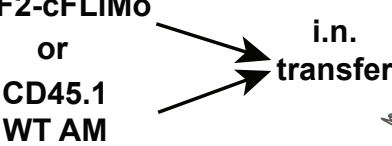

Neonates

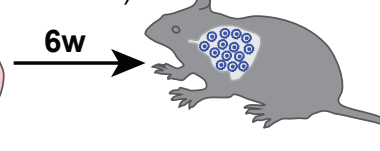

Adults

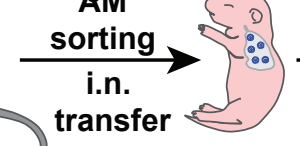

Neonates

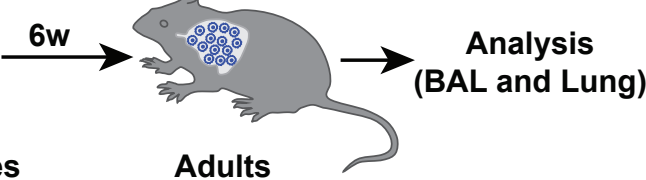

Adults

B

BAL

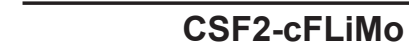

No
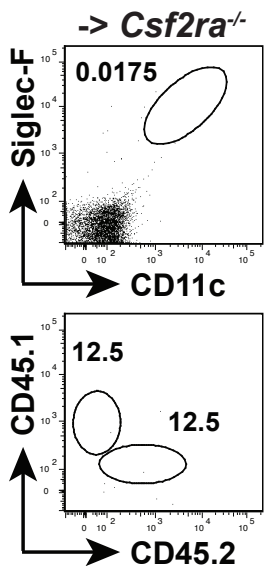

C

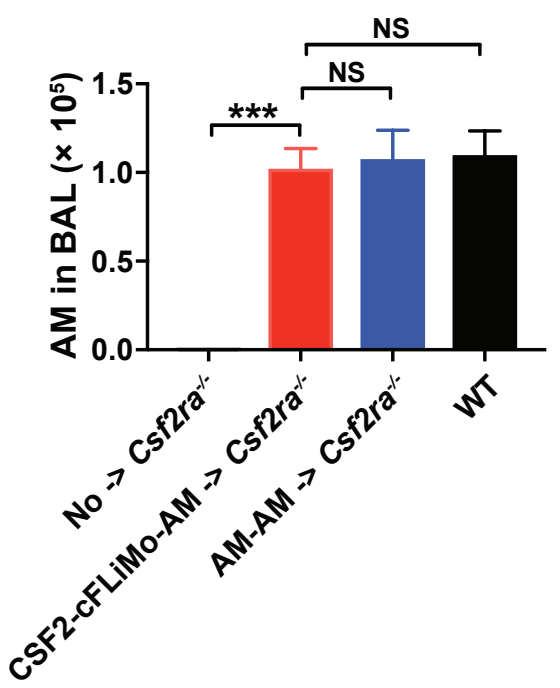

AM-AM WT

$\rightarrow$ Csf2ra ${ }^{-/} \quad$ CD45.2
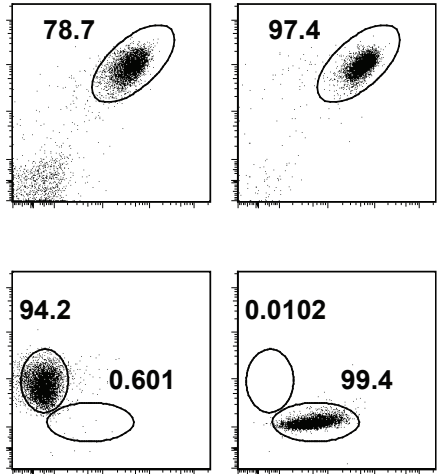

Lung

CSF2-cFLiMo

No -AM
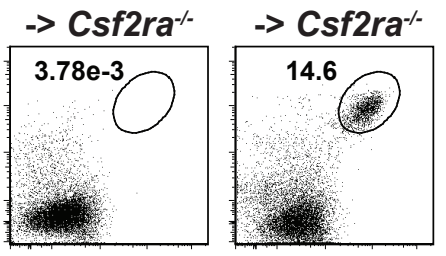

AM-AM

WT

->Csf2ra- CD45.2
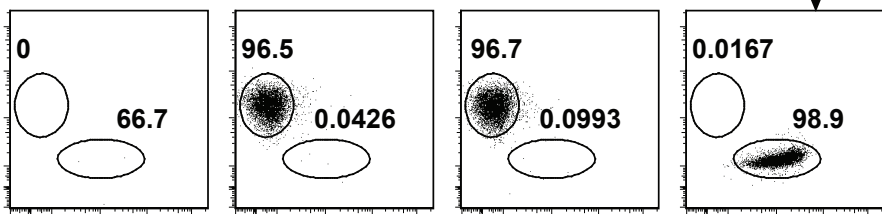

D

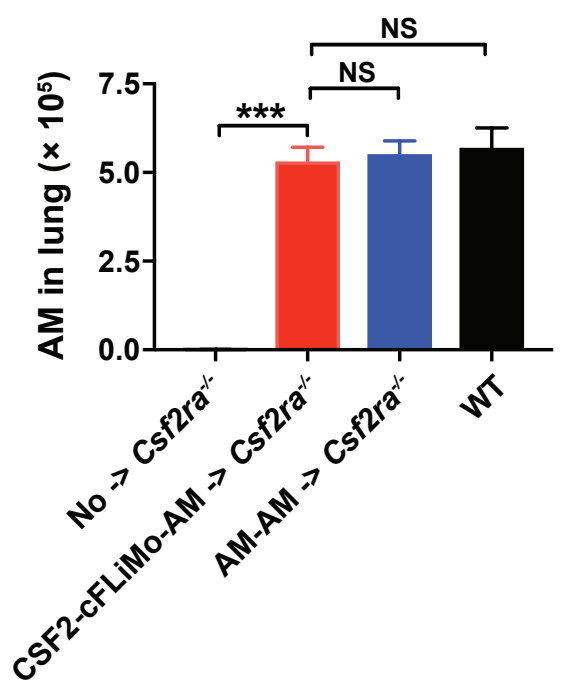




\section{Figure 3}

A

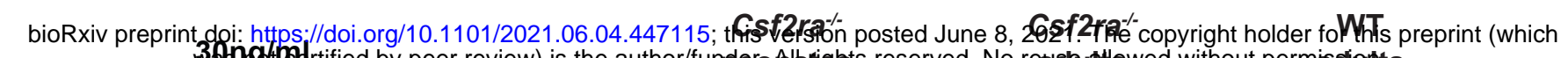

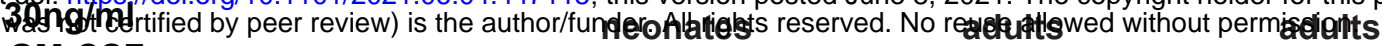

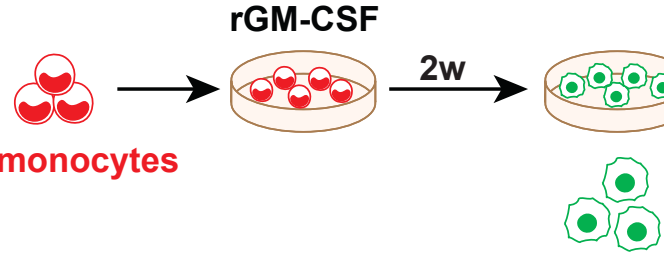

CSF2-cFLiMo

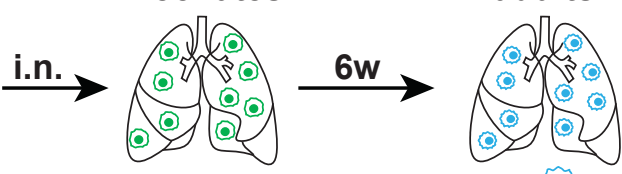

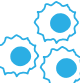

CSF2-cFliMo-AM
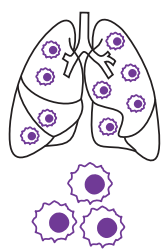

AM
B

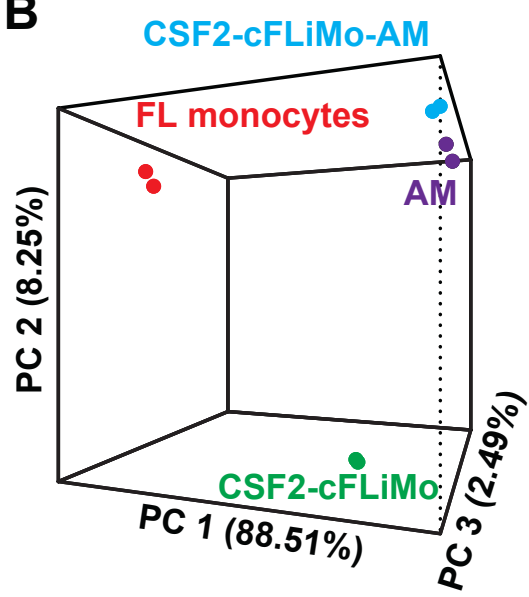

C

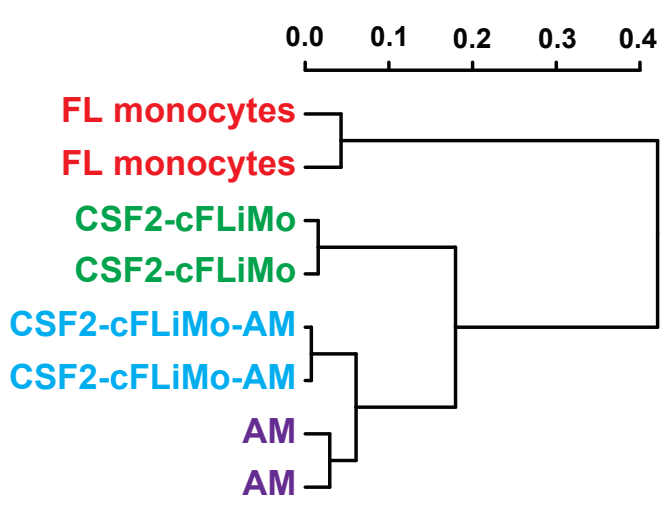

D

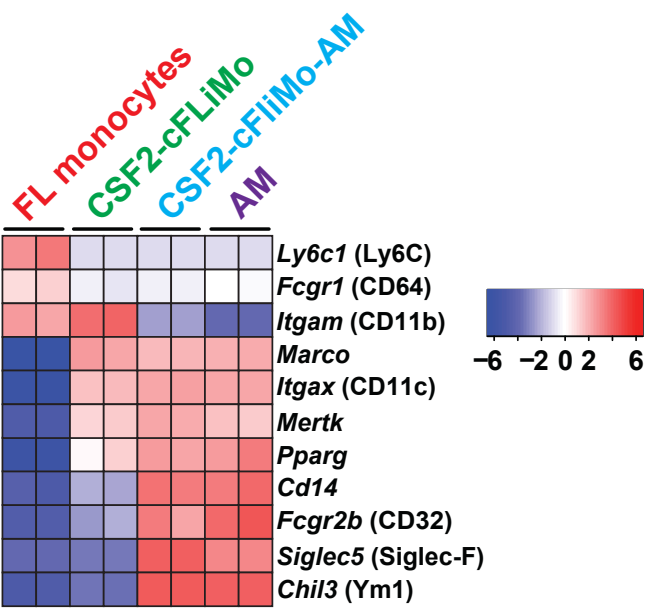

E

FL monocytes CSF2-cFLiMo CSF2-cFliMo-AM

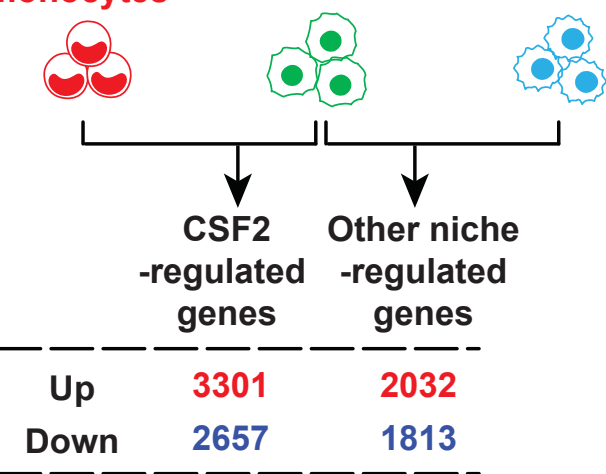

G
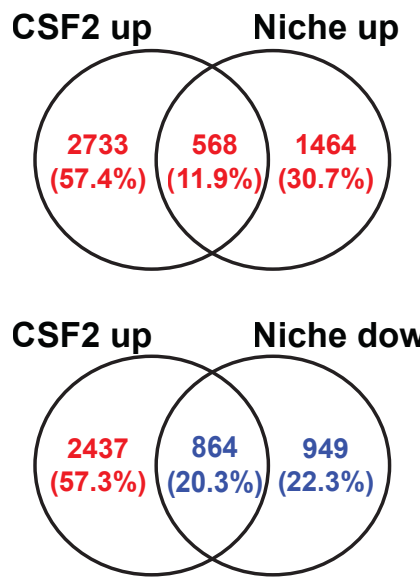
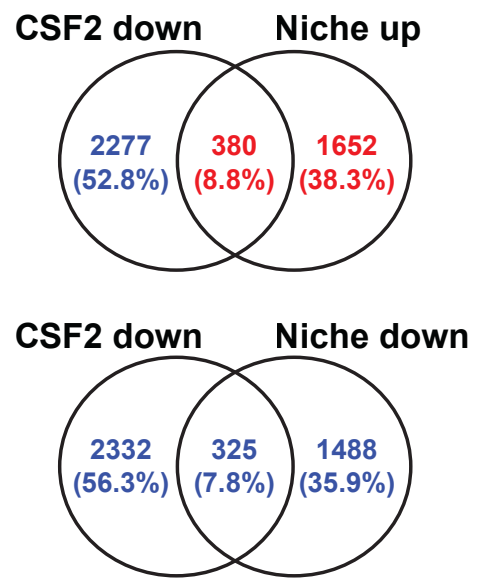

$\mathbf{F}$

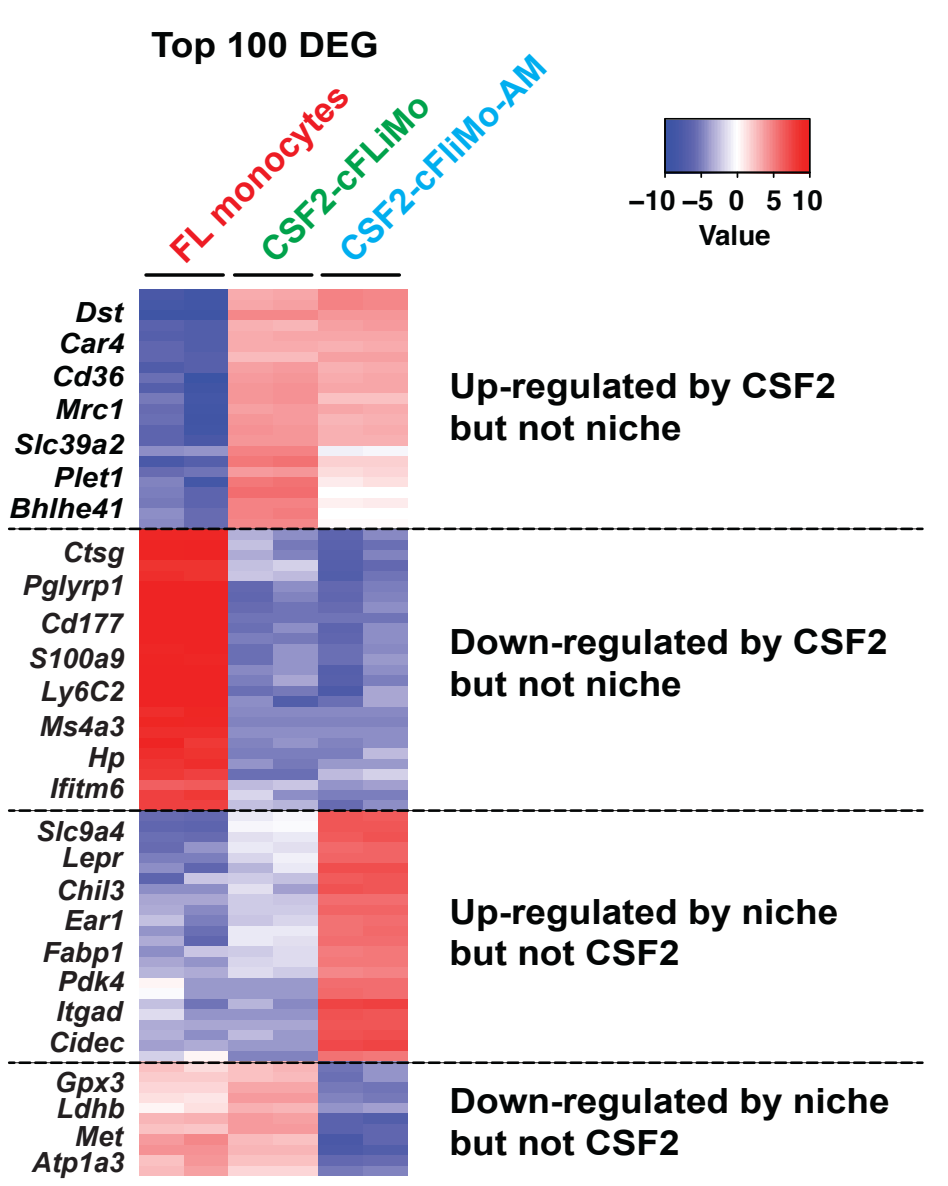




\section{Figure 4}

A

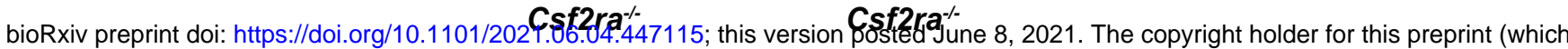
was not certified by peer review) is the author/funder. All rights reserved. No reuse allowed without permission.

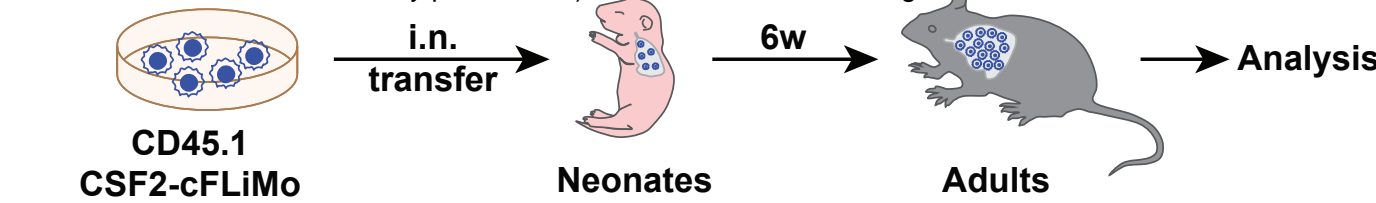

(2w or $4 \mathrm{~m}$ in culture)

B

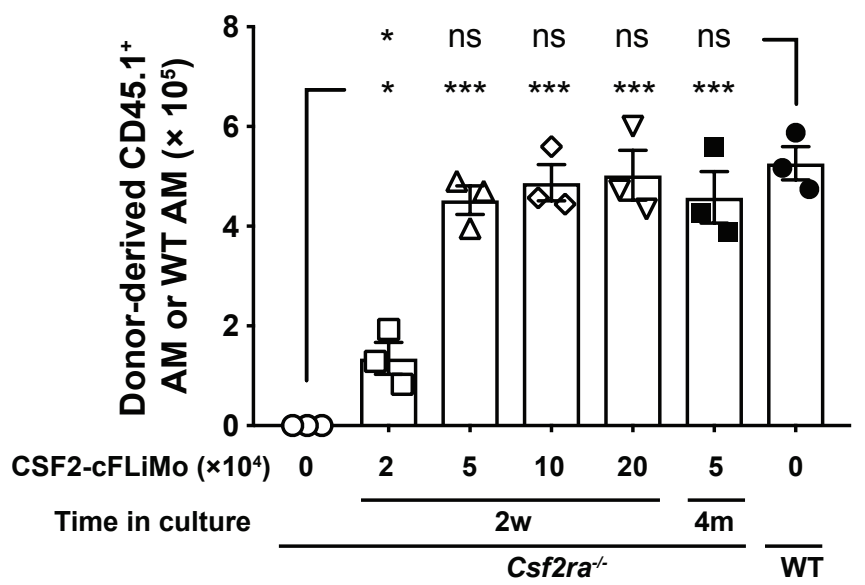

C

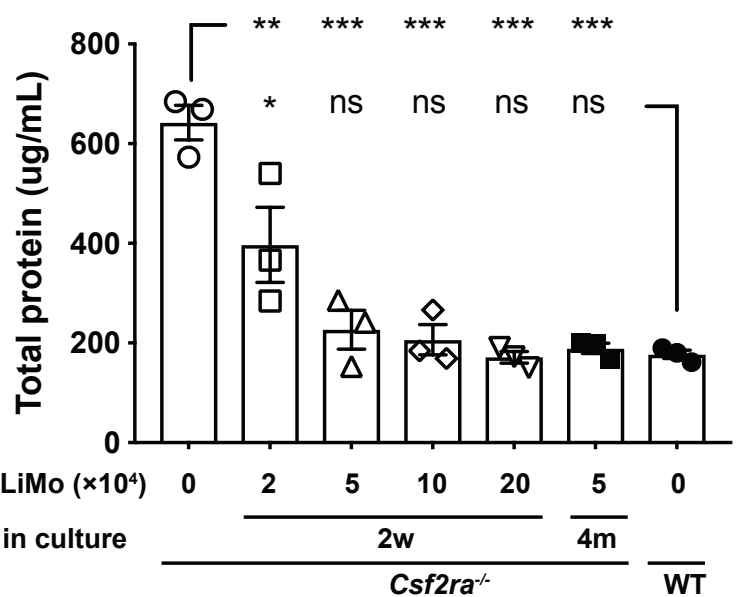

D

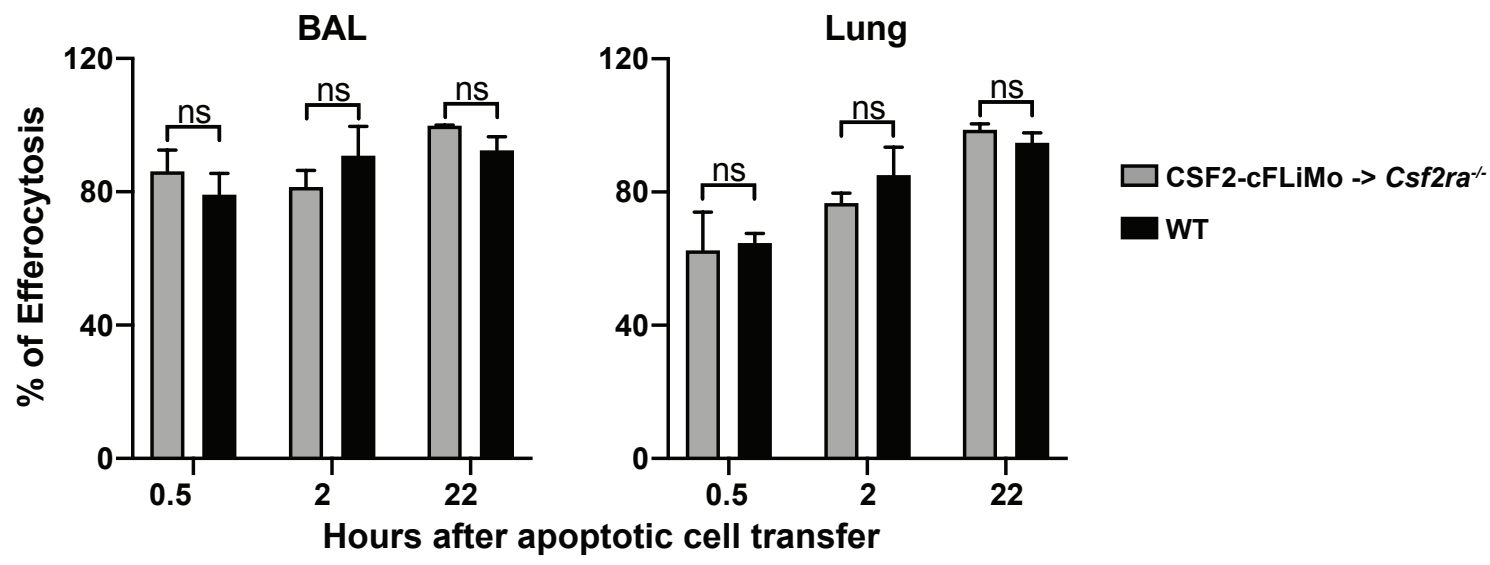

$\mathbf{E}$

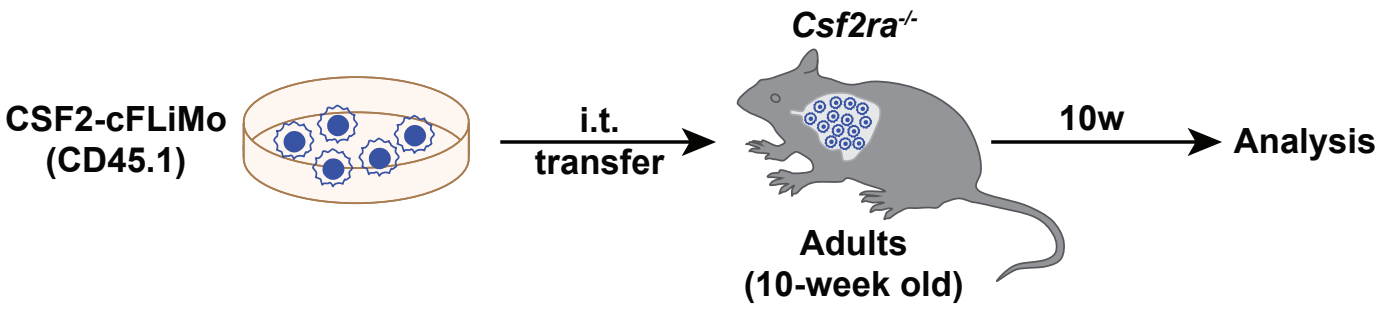

$\mathbf{F}$

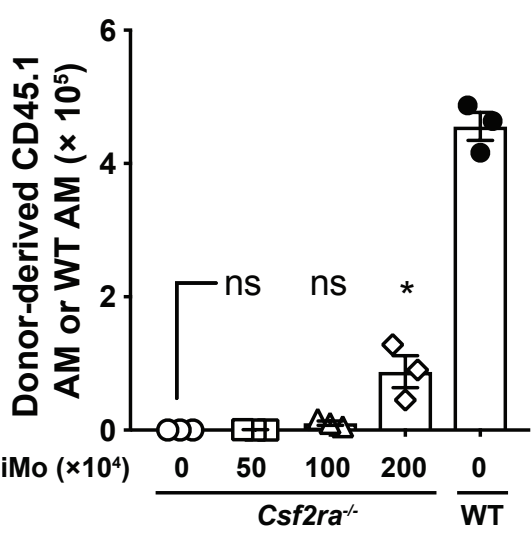

G

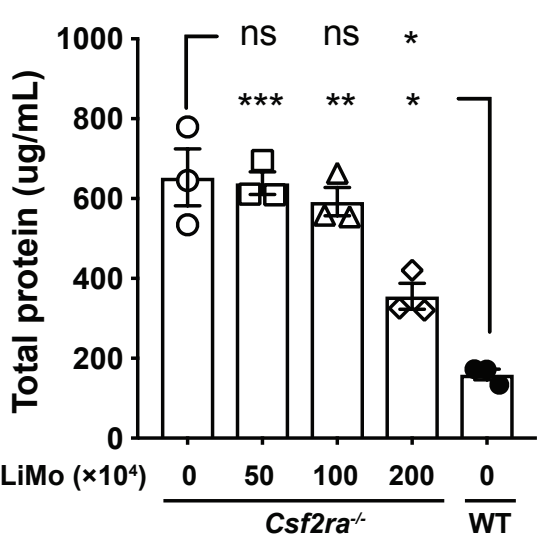




\section{Figure 5}

A

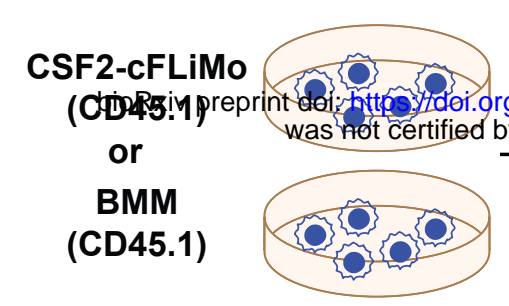

B

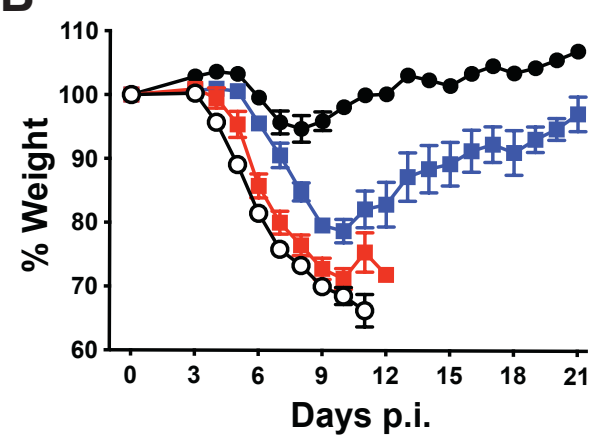

E

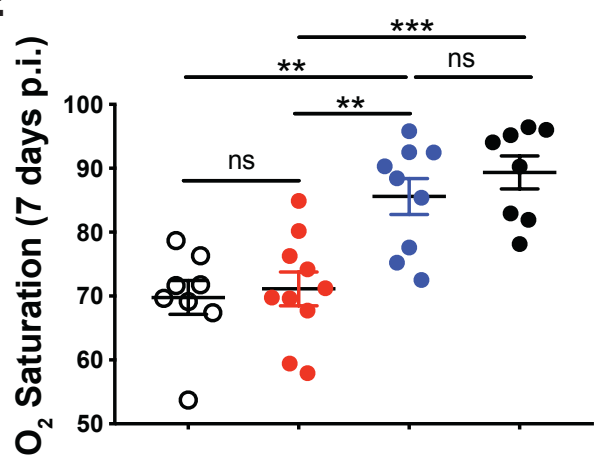

Csf2ra--
Csf2ra

Influenza

$\downarrow$

Csf2ra ${ }^{-/ 2}$

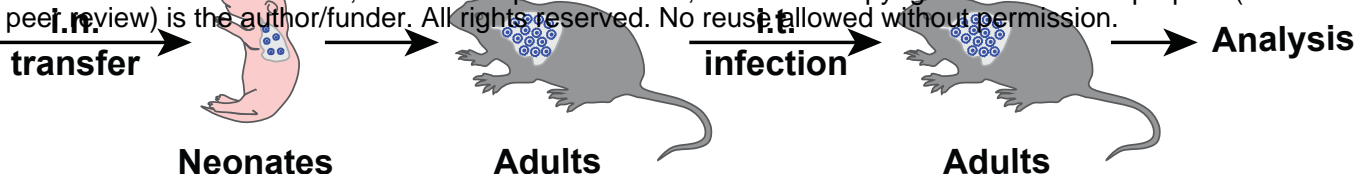

C

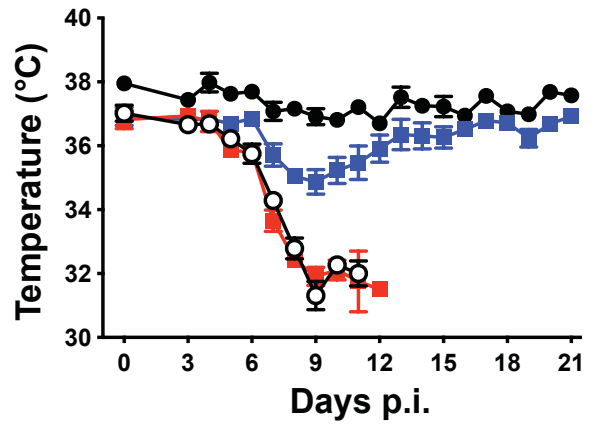

$\mathbf{F}$
D

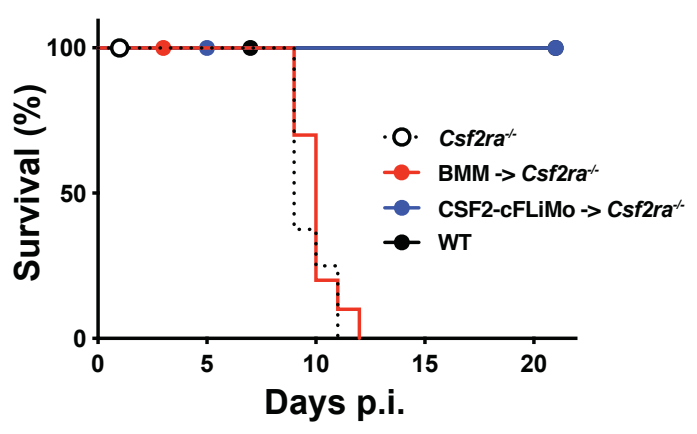

G

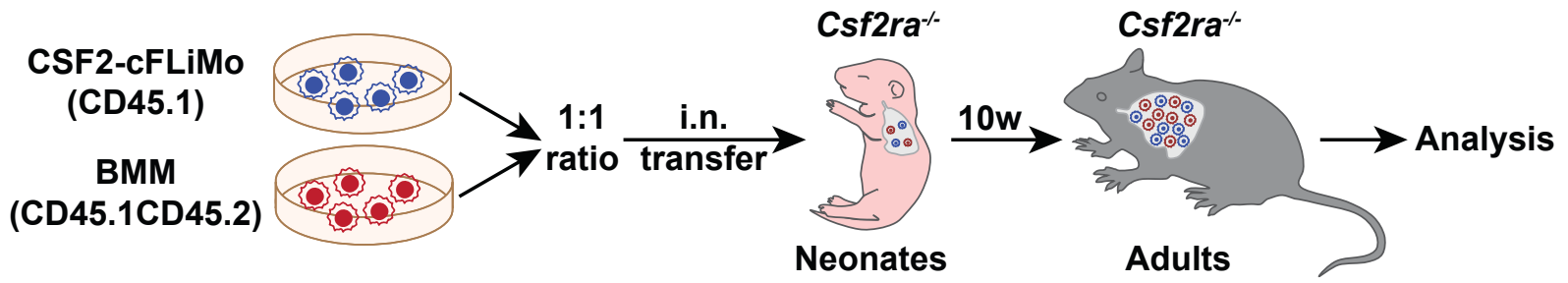

H
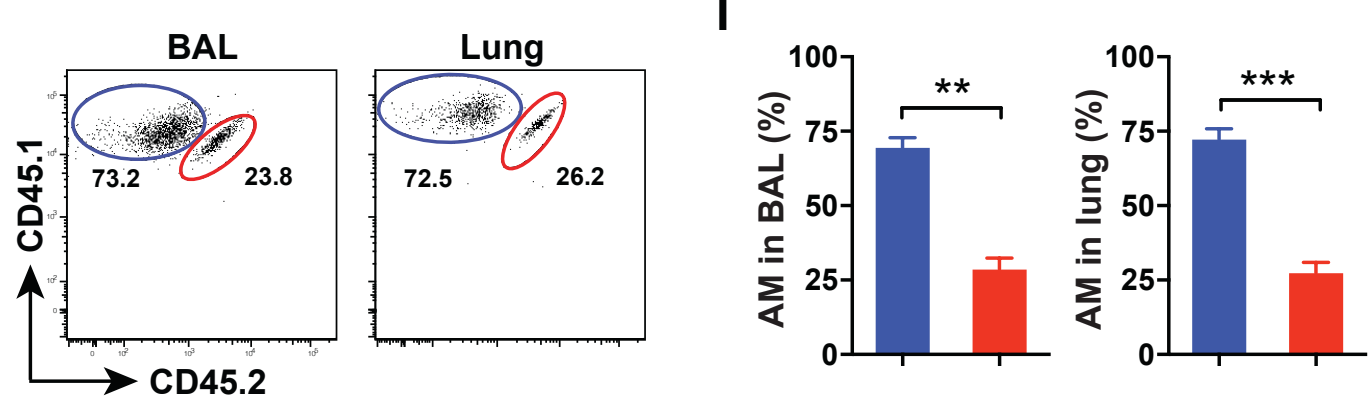

CSF2-cFLiMo (CD45.1)

BMM (CD45.1CD45.2)

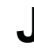

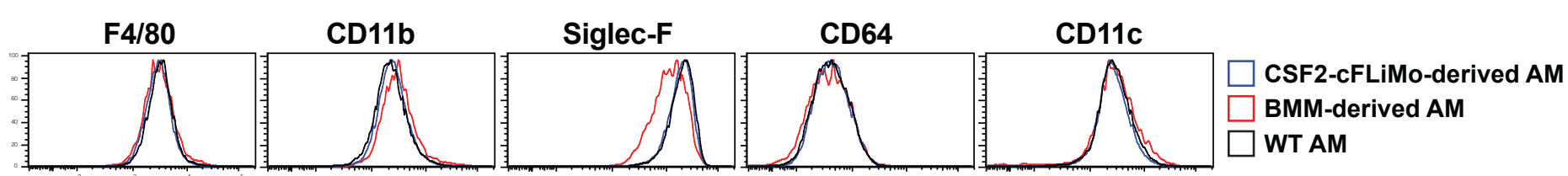

K

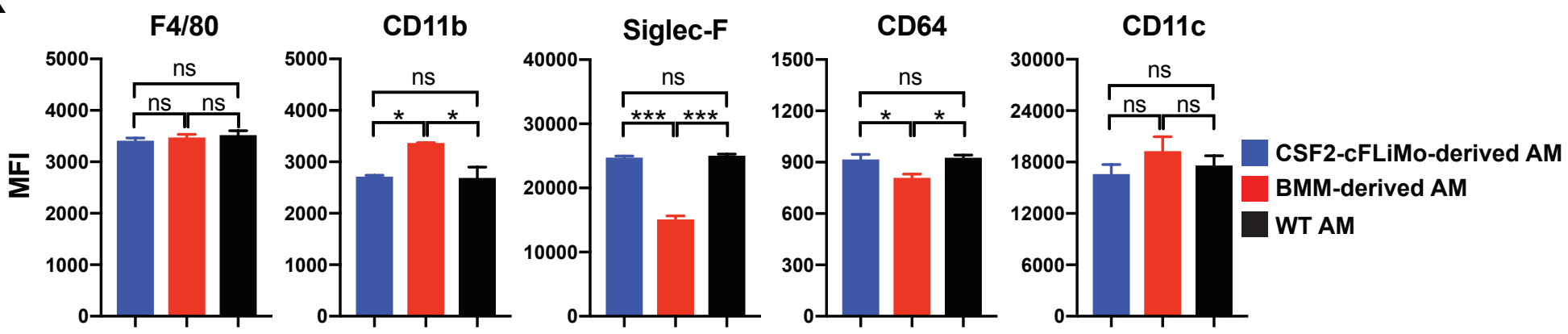




\section{Figure 6}

A bioRxiv preprint doi: https://doi org/10.1111/2021.06.04.447115; this version posted June 8, 2021. The copyright holder for this preprint (which

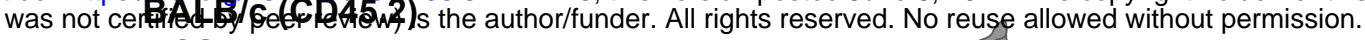

CSF2-cFLiMo

or

BALB/C (CD45.2) : B6 (CD45.1CD45.2) CSF2-CFLiMo $=1: 1$ ratio

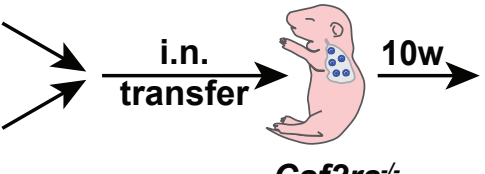

Csf2ra ${ }^{-/}$

Neonates

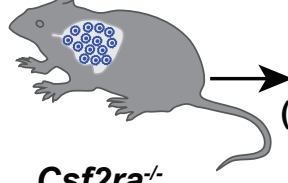

Csf2raAdults
Analysis

(BAL and Lung)
B

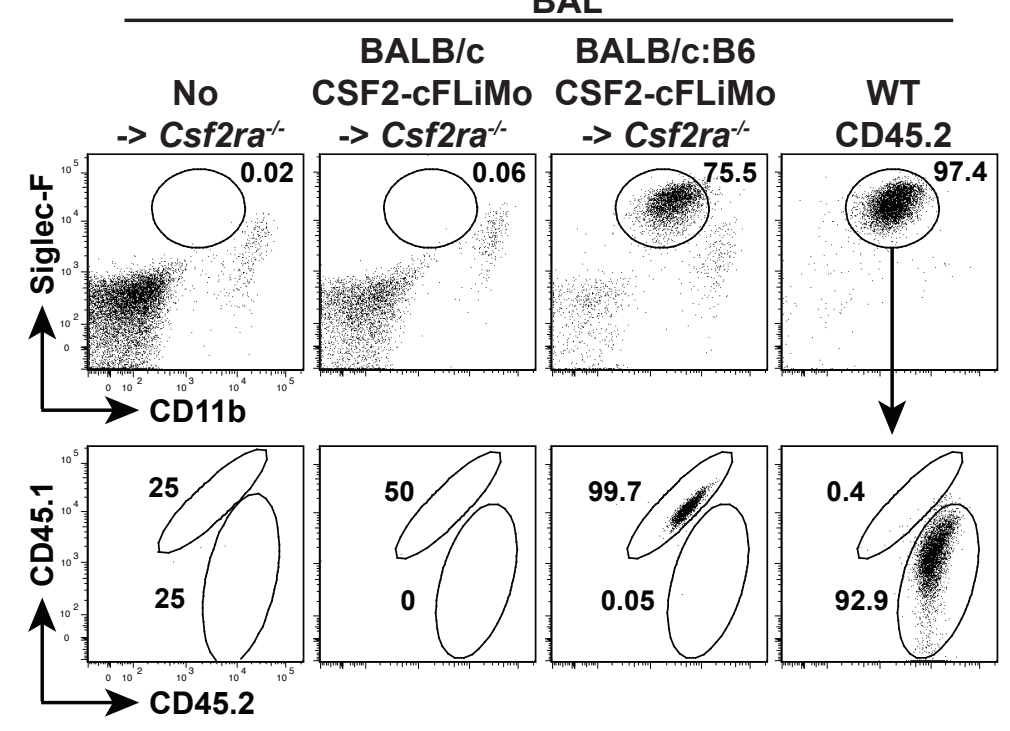

C

\section{BALB/c:B6 co-transfer}

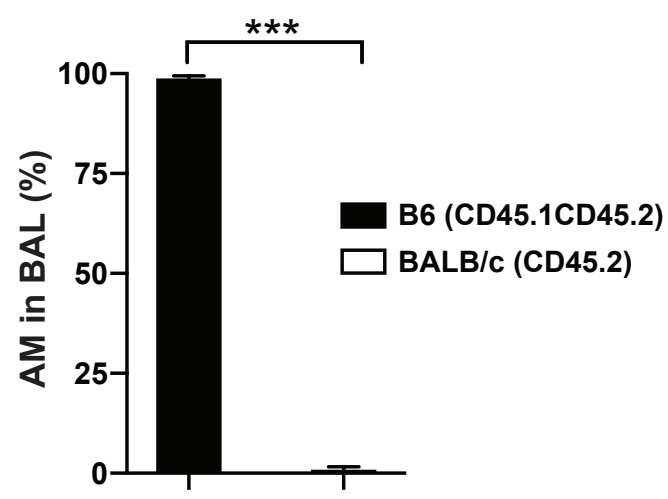

D

\section{CD45.1 Male}

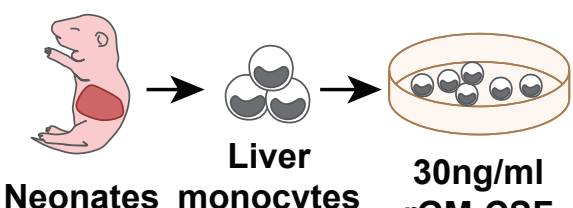

Neonates monocytes

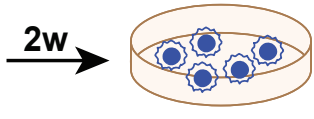

CSF2-cNLiMo i.n.

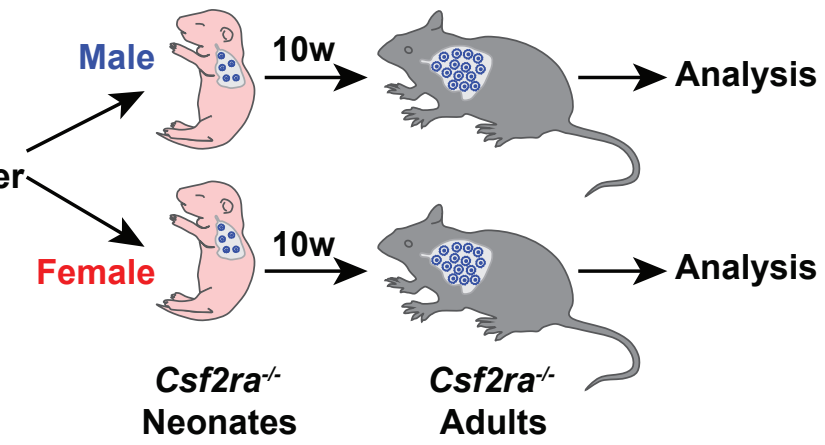

E

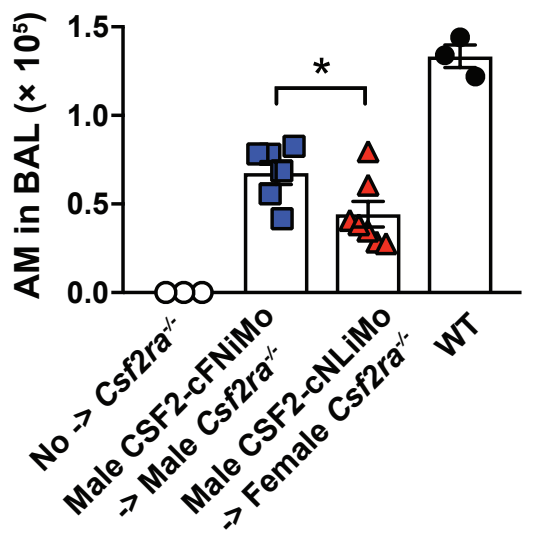

$\mathbf{F}$

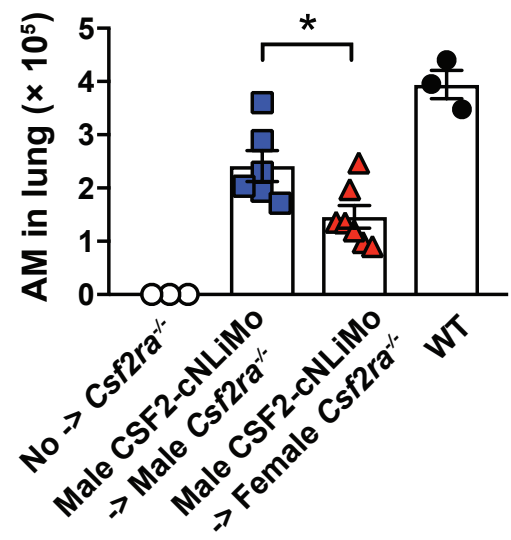




\section{Figure 7}

A

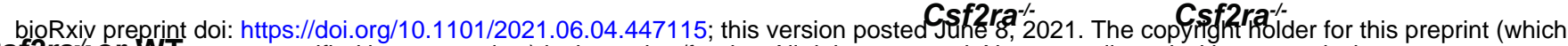
Csf2ra ${ }^{-/}$or WTwas not certified by peer review) is the author/funder. All rights reserved. No reuse allowed without permission.

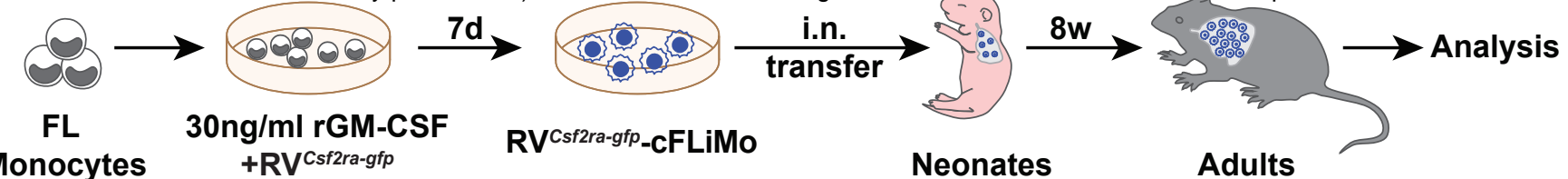

B

Csf2ra ${ }^{-/}$FL Monocytes
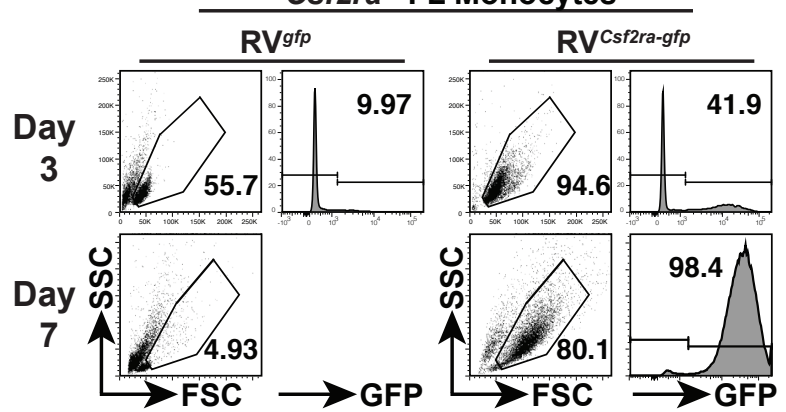

WT FL Monocytes
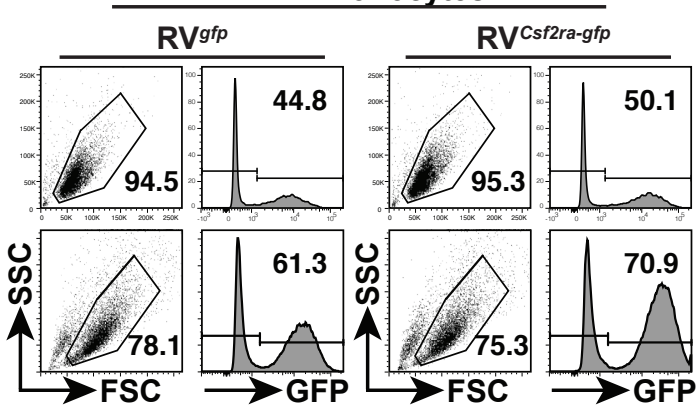

C

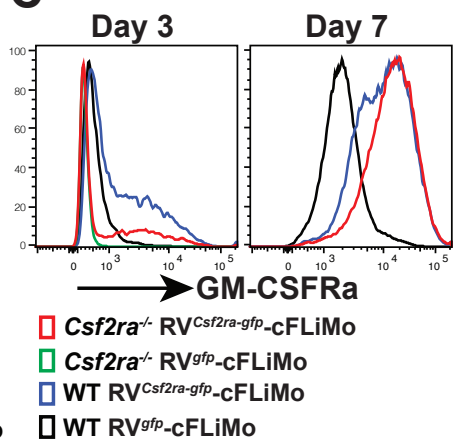

D

BAL
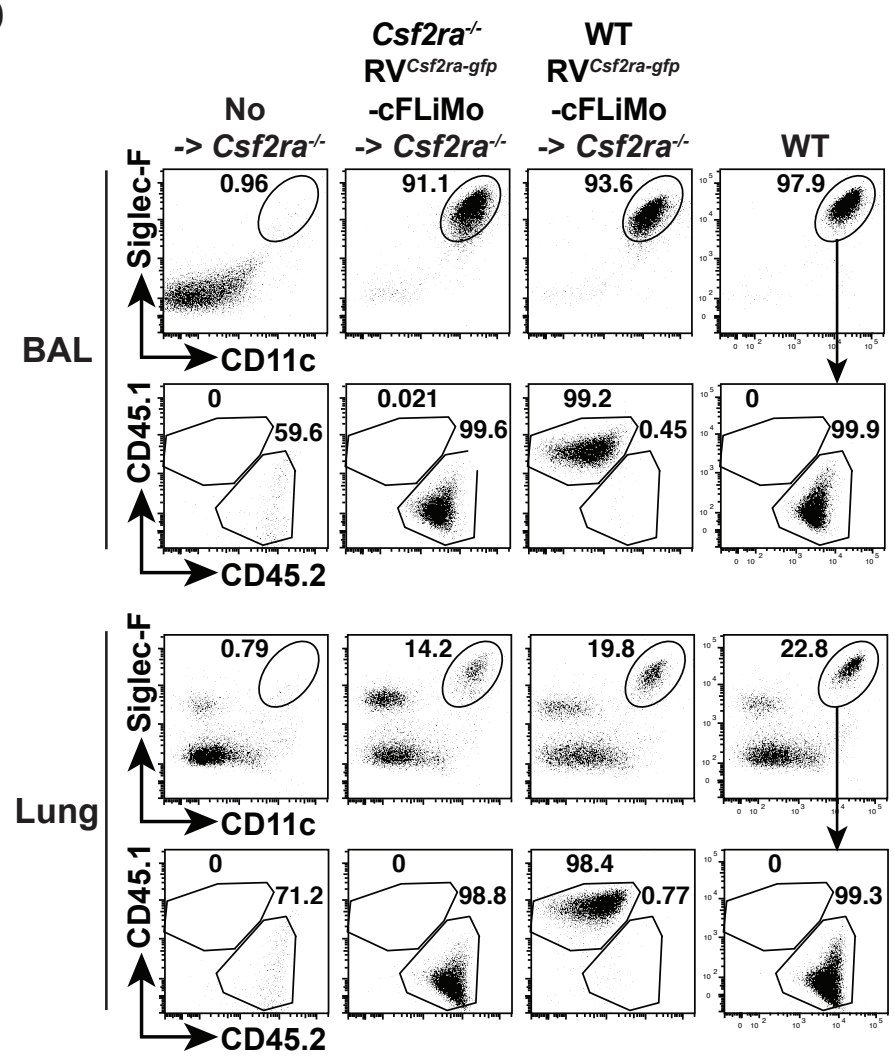

E

F
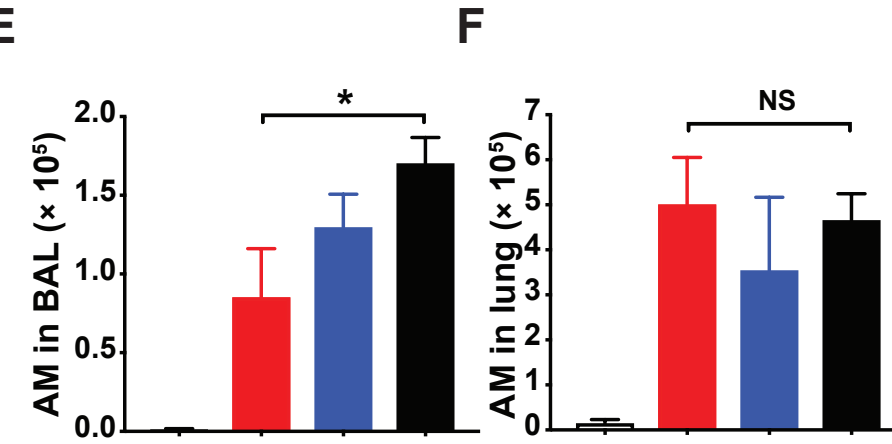

G
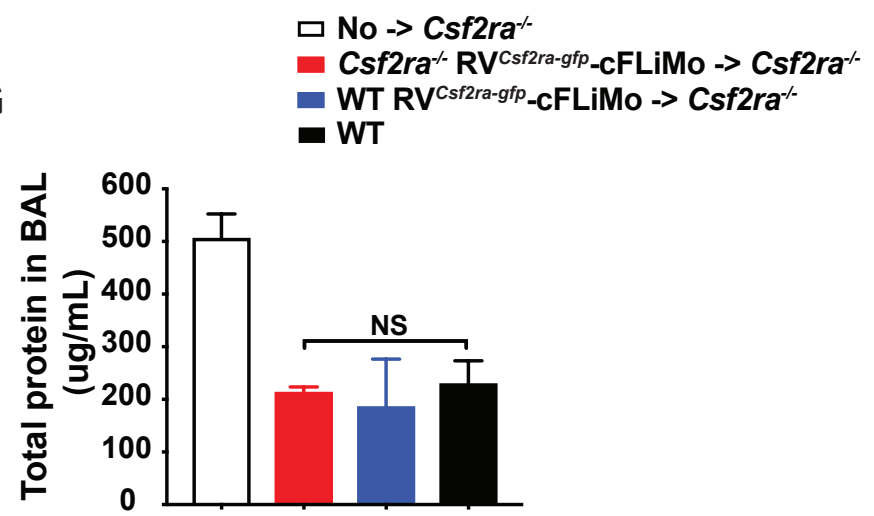

H

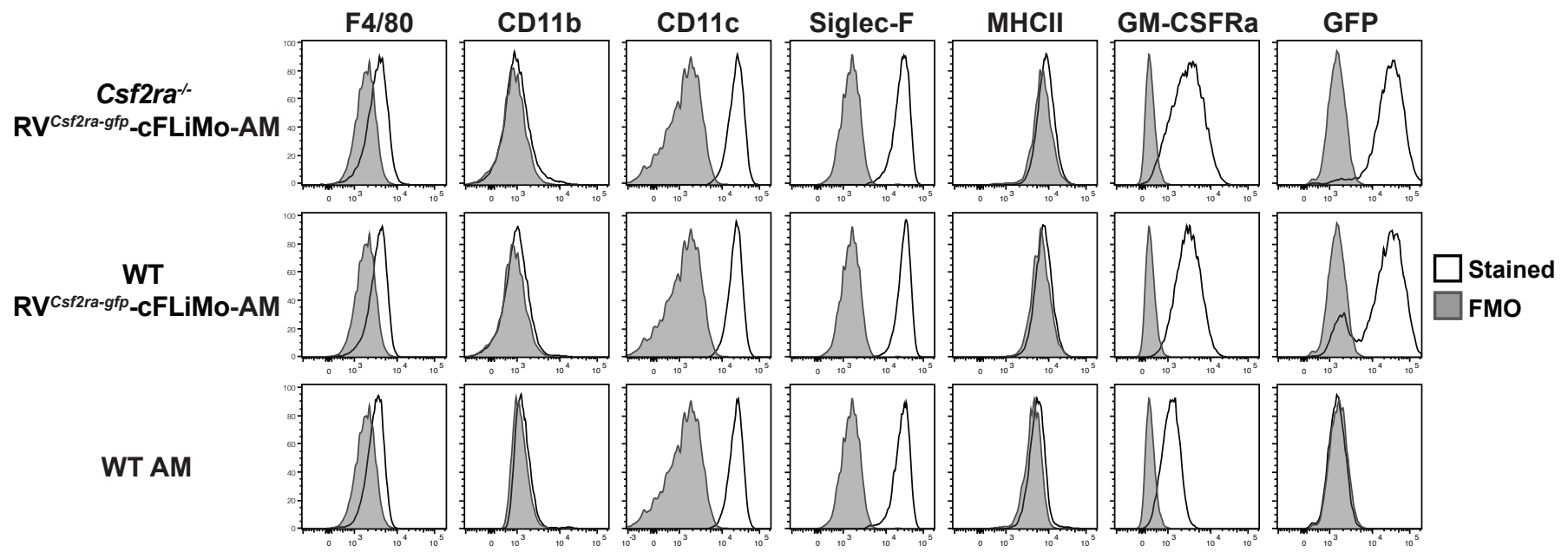

